\title{
Rotational Spring Analogy for Buckling Analysis
}

\author{
B.A. Izzuddin ${ }^{1}$
}

\begin{abstract}
This paper presents a 'rotational spring' analogy which allows the geometric stiffness for a large class of structural problems to be formulated using common notions from linear structural analysis. The proposed analogy offers an intuitive framework which i) sheds significant light on geometric nonlinearity in general, and buckling analysis in particular, ii) explains previous buckling analysis procedures, and iii) is directly applicable to the simplified buckling analysis of discrete as well as continuum structures. The paper discusses the application of the proposed analogy to single degree of freedom, multi-degree of freedom as well as continuous structural systems, providing in the process several illustrative examples. These demonstrate the simplified framework offered by the proposed rotational spring analogy for a wide range of buckling problems, and highlight its conceptual power in furthering the understanding of important issues in geometrically nonlinear analysis.
\end{abstract}

\section{CE DATABASE SUBJECT HEADINGS:}

Buckling; Geometric nonlinearity; Structural analysis; Nonlinear response; Arches; Beam columns; Frames; Plates.

\footnotetext{
${ }^{1}$ Professor of Computational Structural Mechanics, Department of Civil and Environmental Engineering, Imperial College, London SW7 2AZ, United Kingdom. Member, ASCE.
} 


\section{INTRODUCTION}

The buckling of various structural forms attracted significant research interest over many years (e.g. Timoshenko \& Gere, 1961; Nethercot \& Rockey, 1971; Horne, 1975; Allen \& Bulson, 1980; Bradford, 1992), since it normally provides a practical limit on the load carrying capacity of structures. In the context of conservative structural systems, buckling can be characterized by the presence of infinitesimally adjacent equilibrium states at a specific level of loading, where the lowest buckling load is typically of most practical significance.

Theoretical buckling predictions are largely based on conventional principles which consider equilibrium between the applied load and internal forces in the perturbed buckling configuration (Timoshenko \& Gere, 1961; Bažant \& Cedolin, 1991). With this approach, the structural deformations up to the point of buckling are often ignored, and hence the internal forces along the fundamental equilibrium path are assumed to be linearly dependent on the applied load, leading to the expression of buckling as a linear eigenvalue problem. More importantly, this theoretical approach becomes rather impractical for highly indeterminate structures and for continuum structures with irregular geometric domains.

A more general and powerful approach is based on the variational method, or the equivalent principle of virtual work, which is often enhanced with a spatial discretization technique, such as Rayleigh-Ritz or finite element approximation, to deal with continuous problems (Timoshenko \& Gere, 1961; Bažant \& Cedolin, 1991; Crisfield, 1991). As with the theoretical approach, a straightforward expression of buckling as a linear eigenvalue problem is achieved if the pre-buckling deformations are ignored. In the most general case, however, the influence of pre-buckling deformations can be accounted for by tracing the fundamental nonlinear equilibrium path, and establishing the presence of infinitesimally adjacent equilibrium states at various levels of loading. Either way, the buckling condition is most conveniently expressed with this approach in terms of the singularity of the tangent stiffness matrix, which consists of material stiffness and geometric stiffness contributions (Crisfield, 1991; Belytschko et al., 2000). For most common structural forms, buckling occurs when the 
geometric stiffness becomes sufficiently 'negative' to overcome the 'positive' material stiffness, leading to a structure in neutral second-order equilibrium.

When the pre-buckling deformations are ignored and the material response is linear, the material stiffness matrix can be assumed to remain constant, as obtained from linear analysis principles. In fact, even when pre-buckling deformations are significant, the material stiffness matrix can be determined from linear analysis principles, or first-order kinematic descriptions, though these should be applied in the deformed configuration. The geometric stiffness matrix, on the other hand, is determined with the variational approach using secondorder kinematic descriptions, applied in the initial or deformed configurations, depending respectively on whether pre-buckling deformations are negligible or significant. Such higherorder descriptions add a level of complexity to the formulation of the geometric stiffness matrix, and remove the buckling problem from the intuitive notions of linear structural analysis.

This paper proposes a 'rotational spring' analogy which enables the geometric stiffness for a large class of buckling problems to be formulated using first-order kinematic descriptions, thus relying solely on the familiar notions of linear structural analysis. While this analogy is not intended as a replacement to the more rigorous variational approach, it offers a powerful conceptual framework which furthers the understanding of buckling analysis, and provides a simplified approach to the buckling assessment of various structural systems.

The paper proceeds with a brief background on the buckling of discrete structural systems using the variational approach, highlighting the sources of geometric nonlinearity and their reflection in the geometric stiffness. The rotational spring analogy is then developed with reference to axial members, and its application is discussed for single and multi- degree of freedom structural systems. Finally, the proposed analogy is generalized to continuous structural systems, enabling the treatment of a large class of buckling problems, including the buckling of columns, beams, arches and plates. Several illustrative examples are provided throughout, which demonstrate the conceptual power and practical application of the proposed rotational spring analogy. 


\section{BUCKLING OF DISCRETE STRUCTURAL SYSTEMS}

Consider a discrete structural system, with deformations completely defined by a set of discrete freedoms $(\mathbf{U})$, which is subjected to static proportional loads varying according to a common factor $(\lambda)$. Geometrically nonlinear analysis of such a system is typically concerned with establishing states $(\lambda, \mathbf{U})$ which satisfy the conditions of discrete equilibrium in the deformed configuration, as expressed by:

$$
\mathbf{G}=\mathbf{R}-\lambda \mathbf{P}=\mathbf{0}
$$

where $\mathbf{G}=$ out-of-balance forces, $\mathbf{R}=$ structural resistance forces, and $\mathbf{P}=$ unscaled equivalent loads (i.e. equivalent loads at $\lambda=1$ ).

With $\mathbf{R}$, and in the most general case $\mathbf{P}$, determined by $\mathbf{U}$, an equilibrium state $(\lambda, \mathbf{U})$ is identified by the satisfaction of the discrete equilibrium conditions in (1).

For a discrete system consisting of a number of components (e.g. discrete springs, finite elements) with deformations $\mathbf{d}$ and associated internal forces $\mathbf{f}$, it can be shown using the principle of virtual work (Crisfield, 1991) that:

$$
\begin{aligned}
& \mathbf{R}=\mathbf{T}^{\mathrm{T}} \mathbf{f} \\
& \mathbf{T}_{\mathrm{i}, \mathrm{j}}=\frac{\partial \mathbf{d}_{\mathrm{i}}}{\partial \mathbf{U}_{\mathrm{j}}}
\end{aligned}
$$

where $\mathbf{T}=$ transformation matrix, typically dependent on the deformed configuration as defined by $\mathbf{U}$, and obtained from the kinematic relationship expressing $\mathbf{d}$ in terms of $\mathbf{U}$.

Furthermore, $\mathbf{P}$ expresses the variation of the work done by the unscaled loads with respect to $\mathbf{U}$ in the deformed configuration:

$$
\mathbf{P}_{\mathrm{i}}=\frac{\partial W}{\partial \mathbf{U}_{\mathrm{i}}}
$$

where $W=$ work done by the unscaled loads.

For most structural systems, the main source of geometric nonlinearity is due to the nonlinear dependence of $\mathbf{d}$ on $\mathbf{U}$. Another potential source of geometric nonlinearity is due to the nature 
of the loading, specifically when $W$ is nonlinear in terms of $\mathbf{U}$, where for conservative systems $W$ is uniquely determined by $\mathbf{U}$.

Now, buckling analysis of a conservative discrete structural system is typically concerned with establishing one or more critical load factors $\left(\lambda_{c}\right)$ at which infinitesimally adjacent equilibrium states exist for no change in the load factor. That is, two infinitesimally adjacent states $\left(\lambda=\lambda_{c}, \mathbf{U}\right)$ and $\left(\lambda=\lambda_{c}, \mathbf{U}+\delta \mathbf{U}\right)$ exist, where $(\delta \mathbf{U} \neq \mathbf{0})$ represents the buckling mode. Clearly, not only does the critical equilibrium state have to satisfy $(\mathbf{G}=\mathbf{0}$ ) but also ( $\delta \mathbf{G}=\mathbf{0}$ ), leading to the well known eigenvalue problem:

$$
\begin{gathered}
\mathbf{K}_{\mathrm{T}} \delta \mathbf{U}=\mathbf{0} \quad\left(\lambda=\lambda_{\mathrm{c}}, \delta \mathbf{U} \neq \mathbf{0}\right) \\
\mathbf{K}_{\mathrm{Ti}, \mathrm{j}}=\frac{\partial \mathbf{G}_{\mathrm{i}}}{\partial \mathbf{U}_{\mathrm{j}}}=\frac{\partial \mathbf{R}_{\mathrm{i}}}{\partial \mathbf{U}_{\mathrm{j}}}-\lambda \frac{\partial \mathbf{P}_{\mathrm{i}}}{\partial \mathbf{U}_{\mathrm{j}}}
\end{gathered}
$$

where $\mathbf{K}_{\mathrm{T}}=$ tangent stiffness matrix.

Considering (2)-(4), $\mathbf{K}_{\mathrm{T}}$ can be expressed as the sum of a material stiffness matrix $\left(\mathbf{K}_{\mathrm{E}}\right)$ and a geometric stiffness matrix $\left(\mathbf{K}_{\mathrm{G}}\right)$ :

$$
\begin{aligned}
& \mathbf{K}_{\mathrm{T}}=\mathbf{K}_{\mathrm{E}}+\mathbf{K}_{\mathrm{G}} \\
& \mathbf{K}_{\mathrm{E}}=\mathbf{T}^{\mathrm{T}} \mathbf{k}_{\mathrm{e}} \mathbf{T} \\
& \mathbf{K}_{\mathrm{Gi}, \mathrm{j}}=\mathbf{T}^{\mathrm{T}} \mathbf{k}_{\mathrm{g}} \mathbf{T}+\left(\sum_{\mathrm{m}} \frac{\partial^{2} \mathbf{d}_{\mathrm{m}}}{\partial \mathbf{U}_{\mathrm{i}} \partial \mathbf{U}_{\mathrm{j}}}\right)-\lambda \frac{\partial^{2} W}{\partial \mathbf{U}_{\mathrm{i}} \partial \mathbf{U}_{\mathrm{j}}}
\end{aligned}
$$

where, $\mathbf{k}_{\mathrm{e}}+\mathbf{k}_{\mathrm{g}}=\mathbf{k}_{\mathrm{t}}=$ local tangent stiffness matrix of components, given by:

$$
\mathbf{k}_{\mathrm{ti}, \mathrm{j}}=\mathbf{k}_{\mathrm{e} i, \mathrm{j}}+\mathbf{k}_{\mathrm{gi}, \mathrm{j}}=\frac{\partial \mathbf{f}_{\mathrm{i}}}{\partial \mathbf{d}_{\mathrm{j}}}
$$

In establishing the two parts of $\mathbf{k}_{\mathrm{t}}, \mathbf{k}_{\mathrm{e}}$ depends on the tangential material response stiffness, whereas $\mathbf{k}_{\mathrm{g}}$ is the local geometric stiffness matrix which is linearly dependent on the internal forces that equilibrate the applied loading. For a wide range of elastic problems, the local component response can be considered to be linear, in which case $\left(\mathbf{k}_{\mathrm{g}}=\mathbf{0}\right)$ and $\left(\mathbf{k}_{\mathrm{t}}=\mathbf{k}_{\mathrm{e}}=\mathbf{c o n s t}\right)$, though geometric nonlinearity is then manifested on the global structural 
level. Such problems include, for example, discrete structures consisting of linear elastic springs, and more general continuum structures discretized with co-rotational finite elements (Izzuddin, 2001; Izzuddin 2005). Other continuum problems benefit from the use of finite elements that account for local geometric nonlinearity, in which case $\mathbf{k}_{\mathrm{g}}$ becomes a nontrivial linear function of the internal element forces, such as the quartic beam-column element intended to represent the nonlinear response of a whole member (Izzuddin, 1996).

Considering now the global tangent stiffness obtained in (5), it is clear that for a given deformed configuration, as defined by $\mathbf{U}$ and a corresponding compatible $\mathbf{d}, \mathbf{K}_{\mathrm{G}}$ is linearly dependent on the internal forces $\mathbf{f}$ and/or the level of loading as expressed by $\lambda$, but is unrelated to the component material stiffness as defined by $\mathbf{k}_{\mathrm{e}}$. On the other hand, $\mathbf{K}_{\mathrm{E}}$ is strictly related to $\mathbf{k}_{\mathrm{e}}$, where the transformation matrix $\mathbf{T}$ is obtained as in linear structural analysis using first-order kinematic descriptions, only these are applied with reference to the deformed structural geometry. For most structural systems, buckling occurs when $\lambda$ and the corresponding $\mathbf{f}$ lead to a sufficiently 'negative' geometric stiffness $\left(\mathbf{K}_{\mathrm{G}}\right)$ which overcomes the 'positive' material stiffness $\left(\mathbf{K}_{\mathrm{E}}\right)$ resulting in a singular overall tangent stiffness matrix $\left(\mathbf{K}_{\mathrm{T}}\right)$, as required by the eigenvalue problem of (4).

The above approach for determining $\mathbf{K}_{\mathrm{T}}$ at an equilibrium state $(\lambda, \mathbf{U})$ is applicable to all discrete structural systems, including continuum systems which are discretized using finite elements. However, while equilibrium, as expressed by (1)-(3), and $\mathbf{K}_{\mathrm{E}}$, as given in (5.b), are determined by a transformation matrix $\mathbf{T}$ that is identically obtained from linear analysis principles applied to the deformed geometry, $\mathbf{K}_{\mathrm{G}}$ requires a higher-order representation of $\mathbf{d}$ and $W$ in terms of $\mathbf{U}$, thus removing the buckling problem from the intuitive notions of linear structural analysis.

Hereafter, a simplified approach to formulating $\mathbf{K}_{\mathrm{G}}$ is proposed for a large class of conservative small-strain structural problems, in which geometric nonlinearity is associated mainly with transverse deflections to the path of internal axial forces and, possibly, with changes to the orientation of external applied forces. This approach is based on an analogy which enables $\mathbf{K}_{\mathrm{G}}$ to be determined from equivalent rotational springs using the familiar 
principles of linear structural analysis, hence the term 'rotational spring analogy'. It should be noted that the proposed approach is not intended as a replacement to the more rigorous formulation encapsulated by (5.c), which can deal with the full spectrum of geometrically nonlinear problems including, for example, nonlinearity arising from an applied conservative moment with a rotating axis. It does, however, offer an intuitive framework which i) sheds significant light on geometric nonlinearity in general, and buckling analysis in particular, ii) explains previous buckling analysis procedures, and iii) is directly applicable to the simplified buckling analysis of discrete as well as continuum structures.

Perhaps it is helpful to emphasize here that the proposed method for buckling analysis, which employs common notions from linear structural analysis, does not imply a linearization of the nonlinear structural response, since buckling is an inherently nonlinear response phenomenon. Clearly, the mere consideration in this method of $\mathbf{K}_{\mathrm{G}}$, which depends on the internal forces and level of loading, is a reflection of geometric nonlinearity in the structural response. However, a major contention of this work is that $\mathbf{K}_{\mathrm{G}}$ can be easily determined using the familiar principles of linear structural analysis based on first-order kinematic descriptions.

It is finally noted that since the proposed approach is concerned with the simplified formulation of $\mathbf{K}_{\mathrm{G}}$ only, focus is given here to the geometrically nonlinear response in the context of structures with a linear material response, for which $\mathbf{K}_{\mathrm{E}}$ is independent of the internal force/deformation state. Notwithstanding, the proposed analogy can be equally used to obtain $\mathbf{K}_{\mathrm{G}}$ for structures with material nonlinearity, though the feasibility of expressing elasto-plastic structural buckling as a conventional eigenvalue problem is a much wider question, since $\mathbf{K}_{\mathrm{E}}$ is not uniquely defined in the plastic range due to different loading/unloading material stiffnesses.

\section{ROTATIONAL SPRING ANALOGY}

As discussed in the previous section, the geometric stiffness matrix associated with a known equilibrium state derives from the internal component forces and, possibly, from the applied external loads, considering permissible perturbations to the structural geometry from the 
current configuration. For practical structural engineering problems, component strains may be assumed to remain relatively small, and hence the dominant change in geometry required for determining the geometric stiffness is related to rigid body modes, specifically rigid body rotations, of individual elements. This is particularly true in the limit as the number of elements used for representing a whole member is increased, as elaborated in sub-section "Bending Element".

Hereafter, the proposed rotational spring analogy is discussed with reference to discrete onedimensional elements with internal axial forces and subjected to applied forces at the ends. Therefore, elements with significant internal bending moments, applied external moments or distributed loads are excluded from this initial discussion, though the proposed analogy is generalized in section "Continuous Systems" to deal with the first and last of these exclusions.

\section{Straight Element}

Consider an element which remains straight, as shown in Figure 1, subject to an axial load at the ends that is independent of structural deformation. Clearly, the only first-order change in forces required to maintain equilibrium under an infinitesimal rigid body rotation $(\delta \rho)$ is represented by a couple proportional to the internal axial force $(\mathrm{F})$ and the element length (L). This geometric stiffness is clearly equivalent to a rotational spring with a stiffness given simply as:

$$
\mathrm{k}_{\rho}=\mathrm{FL}
$$

It is worth noting that the equivalent rotational spring is stabilizing $\left(\mathrm{k}_{\rho}>0\right)$ for a tensile axial force $(\mathrm{F}>0)$ and is conversely destabilizing $\left(\mathrm{k}_{\rho}<0\right)$ for a compressive axial force $(\mathrm{F}<0)$. Furthermore, in the general case including in-plane and out-of-plane rotations, two corresponding rotational springs are required with the same stiffness as given by (7).

\section{Bending Element}

Considering now an axially loaded element which may bend due to buckling, as depicted in Figure 2, the equivalent rotational springs, generalizing from the straight element case, are 
uniformly distributed along the element length with a rotational stiffness per unit length equal to the internal axial force $(\mathrm{F})$. This effect can be shown to be equivalent to a single discrete rotational spring connected to the element chord with a stiffness given by (7), in addition to distributed rotational springs connecting the element chord to the deformed element reference line with a stiffness:

$$
\mathrm{k}_{\theta}=\mathrm{F}
$$

Clearly, a bending element presents an increased geometric stiffness, both stabilizing and destabilizing, in comparison with a straight element, due to the additional 'local' rotational springs $\left(\mathrm{k}_{\theta}\right)$. Notably, the global and local rotational springs, $\mathrm{k}_{\rho}$ and $\mathrm{k}_{\theta}$, which are respectively associated with the element rigid body rotation and strain-inducing deformations, are also respectively responsible for the commonly known P- $\Delta$ and P- $\delta$ effects (Chan \& Zhou, 2000). The relative importance of global and local geometric nonlinearity for an element is obviously related to the relative values of $\delta \rho$ and $\delta \theta$ (note that $\delta \theta$ varies along the element length), which depend on the specific buckling mode and the number of elements per member. Considering Figure 3, it is evident that the importance of local geometric nonlinearity, and hence the significance of strain-inducing modes for the geometric stiffness, diminishes as the number of elements is increased.

\section{Radial Loads}

When the geometric stiffness is determined from element contributions as discussed above, no additional contributions arise from conservative loads consisting of forces that are independent of structural deformation, both in terms of magnitude and direction. One type of conservative loading which contributes additional geometric stiffness, however, is an applied radial force, where only the direction is dependent on structural deformation such that the force always points towards a fixed centre of rotation (Bažant \& Cedolin, 1991). As illustrated in Figure 4, and following a similar argument as in sub-section "Straight Element", the geometric stiffness arising from a radial load $(\mathrm{P})$ can be represented by an equivalent rotational spring with a stiffness given by: 


$$
\mathrm{k}_{\rho}=\mathrm{PR}
$$

where $\mathrm{R}$ = radial distance between the point of load application and the centre of rotation. Interestingly, this contribution is stabilizing for a radial load pointing towards the centre $(\mathrm{P}>0)$ and is conversely destabilizing for a load pointing away from the centre $(\mathrm{P}<0)$. Therefore, if the applied load is fully resisted by a single straight element $(\mathrm{F}=-\mathrm{P})$ with an identical centre of rotation to that of the radial force $(L=R)$, the overall effect on the geometric stiffness, as obtained from (7) and (9), is neutral.

\section{SINGLE DEGREE OF FREEDOM SYSTEMS}

Consider a single degree of freedom (SDOF) system consisting of straight elements with/without discrete springs such that the deformed configuration, or at least the buckling mode of interest, is uniquely defined in terms of a single displacement parameter (U). For a known equilibrium state, the single-term material stiffness may be readily obtained from (5.b), whereas the single-term geometric stiffness can be determined from the rotational spring analogy using similar linear analysis principles as follows:

$$
\mathrm{K}_{\mathrm{G}}=\mathbf{T}_{\rho}^{\mathrm{T}} \operatorname{diag}\left(\mathbf{k}_{\rho}\right) \mathbf{T}_{\rho}=\sum_{\mathrm{i}=1}^{\mathrm{n}} \mathbf{k}_{\rho \mathrm{i}} \mathbf{T}_{\rho \mathrm{i}}^{2}
$$

where, $\operatorname{diag}\left(\mathbf{k}_{\rho}\right)=$ diagonal stiffness matrix associated with the $\mathrm{n}$ equivalent rotational springs, and $\mathbf{T}_{\rho}=$ transformation vector expressing a first-order kinematic approximation of the equivalent spring rotations $(\rho)$ in terms of the single parameter $(U)$ according to:

$$
\rho=\mathbf{T}_{\rho} \mathrm{U}
$$

Two examples are provided hereafter relating to buckling analysis of SDOF systems that consist of straight elements. It is noted that the case of bending elements, which is not considered by (10)-(11), is dealt with in section "Continuous Systems".

\section{Laterally Restrained Axial Member}

Consider a compressive member consisting of two pin-ended elements with a flexible midlength lateral restraint, as shown in Figure 5. Although this is not strictly a SDOF problem, unless the axial elements are fully rigid, the buckling mode for straight elements can be 
represented by a single parameter U. Assuming that the element lengths are unaffected by $\mathrm{P}$, and observing the first-order kinematic expressions for $\rho$ and the stiffness of the two equivalent rotational springs in Figure 5, the geometric stiffness associated with $U$ is obtained from (10)-(11) as:

$$
\mathrm{K}_{\mathrm{G}}=\sum_{\mathrm{i}=1}^{2} \mathbf{k}_{\rho \mathrm{i}}\left(\frac{\boldsymbol{\rho}_{\mathrm{1}}}{\mathrm{U}}\right)^{2}=-\frac{2 \mathrm{P}}{\mathrm{L}}
$$

On the other hand, the material stiffness associated with $U$ is simply given by:

$$
\mathrm{K}_{\mathrm{E}}=\mathrm{k}
$$

Buckling occurs when the overall tangent stiffness is zero, leading to the following critical load:

$$
\mathrm{K}_{\mathrm{T}}=\mathrm{K}_{\mathrm{E}}+\mathrm{K}_{\mathrm{G}}=0 \Rightarrow \mathrm{P}_{\mathrm{c}}=\frac{\mathrm{kL}}{2}
$$

which is identical to the prediction of second-order structural analysis.

\section{Rigid Bar System with Prestressed Cables}

Consider the planar SDOF structural system shown in Figure 6, which consists of three rigid bars and two relatively flexible cables prestressed with an axial force T. Assuming first that the cable stiffness is negligible, a zero material stiffness $\left(\mathrm{K}_{\mathrm{E}}\right)$ is obviously obtained. However, because of the cable prestress, the geometric stiffness $\left(\mathrm{K}_{\mathrm{G}}\right)$ is initially positive, and buckling occurs under the applied loads $(\mathrm{P})$ when $\mathrm{K}_{\mathrm{G}}$ becomes zero. Although this is not a typical structural buckling problem, it is characteristic of more advanced structural applications, such as foldable structures.

With the cable stiffness assumed negligible, the cable axial forces remain constant at $\mathrm{T}$, and all the loading is transferred to the bars. Up to the buckling point, and because of symmetry, the structure sustains the applied loads without a change in geometry, leading to a trivial fundamental equilibrium path, where the axial forces in the bars can be readily obtained from $\mathrm{T}$ and $\mathrm{P}$. Considering the stiffnesses of the five equivalent rotational springs and the first- 
order kinematic expressions for $\rho$ in Figure 6, the geometric stiffness associated with the single rotational freedom $(\mathrm{U})$ is obtained from (10)-(11) as:

$$
\mathrm{K}_{\mathrm{G}}=\sum_{\mathrm{i}=1}^{5} \mathbf{k}_{\rho \mathrm{i}}\left(\frac{\boldsymbol{\rho}_{\mathrm{I}}}{\mathrm{U}}\right)^{2}=2.4425 \mathrm{TL}-2.6354 \mathrm{PL}
$$

Buckling occurs when the overall tangent stiffness, or in this case the geometric stiffness, becomes zero, leading to the following critical load:

$$
\mathrm{K}_{\mathrm{T}}=\mathrm{K}_{\mathrm{E}}+\mathrm{K}_{\mathrm{G}}=\mathrm{K}_{\mathrm{G}}=0 \Rightarrow \mathrm{P}_{\mathrm{c}}=0.9268 \mathrm{~T}
$$

Of course, a more realistic assessment would include the cable stiffness, but this only affects $\mathrm{K}_{\mathrm{E}}$, which can be easily determined using linear analysis principles as expressed by (5.b). This would in turn increase the critical load, which can still be obtained from (16), but considering the non-zero $\mathrm{K}_{\mathrm{E}}$ with $\mathrm{K}_{\mathrm{G}}$ as obtained in (15).

Finally, this problem demonstrates the conceptual power and practical benefits of the proposed rotational spring analogy, which provides a simple means to obtain the buckling load using linear analysis principles. Clearly, the application of conventional buckling analysis based on equilibrium in the perturbed configuration becomes rather complex for such a problem.

\section{MULTI-DEGREE OF FREEDOM SYSTEMS}

For a multi-degree of freedom system (MDOF) in which the deformed configuration, or at least the buckling mode of interest, is uniquely defined by several displacement parameters (U), the same linear analysis principles employed for the SDOF case can be used to determine the geometric stiffness matrix from the equivalent rotational springs:

$$
\mathbf{K}_{\mathrm{G}}=\mathbf{T}_{\rho}^{\mathrm{T}} \operatorname{diag}\left(\mathbf{k}_{\rho}\right) \mathbf{T}_{\rho}
$$

where now $\mathbf{T}_{\rho}=$ transformation matrix expressing a first-order kinematic approximation of $\boldsymbol{\rho}$ in terms of $\mathbf{U}$ according to:

$$
\rho=\mathbf{T}_{\rho} \mathbf{U}
$$


The buckling condition of (4.a) can now be checked considering $\mathbf{K}_{\mathrm{T}}$ of (5.a), where $\mathbf{K}_{\mathrm{E}}$ may be determined from (5.b), while $\mathbf{K}_{\mathrm{G}}$ is obtained from the rotational spring analogy according to (17).

For systems with a linear elastic material response and where the deformations up to the point of buckling are relatively small, $\mathbf{K}_{\mathrm{E}}$ can be assumed constant and $\mathbf{K}_{\mathrm{G}}$ becomes linearly dependent on the applied loading, and hence the buckling problem can be conveniently expressed as a linear eigenvalue problem in terms of the load factor. However, although linear eigenvalue analysis is well established, sophisticated procedures and significant computational demand are often required for large MDOF systems. Fortunately, very good estimates of the lowest buckling load may be obtained efficiently using problem-reduction, similar to Rayleigh-Ritz approximation (Bažant \& Cedolin, 1991), where a reduced set of deformation modes is used to approximate the actual buckling mode:

$$
\mathbf{U}=\Phi \mathbf{u}
$$

in which $\boldsymbol{\Phi}=$ matrix of approximation deformation modes, and $\mathbf{u}=$ reduced parameters.

The reduced eigenvalue problem can then be expressed as:

$$
\mathbf{k}_{\mathrm{T}} \delta \mathbf{u}=\mathbf{0} \quad(\delta \mathbf{u} \neq \mathbf{0})
$$

where,

$$
\begin{aligned}
& \mathbf{k}_{\mathrm{T}}=\mathbf{k}_{\mathrm{E}}+\mathbf{k}_{\mathrm{G}} \\
& \mathbf{k}_{\mathrm{E}}=\boldsymbol{\Phi}^{\mathrm{T}} \mathbf{K}_{\mathrm{E}} \boldsymbol{\Phi} \\
& \mathbf{k}_{\mathrm{G}}=\boldsymbol{\Phi}^{\mathrm{T}} \mathbf{K}_{\mathrm{G}} \boldsymbol{\Phi}
\end{aligned}
$$

It can be easily shown that the lowest buckling load obtained from a reduced linear eigenvalue problem, as expressed by (20) and (21), overestimates the actual lowest buckling load of the original MDOF problem (Bažant \& Cedolin, 1991). Naturally, the accuracy of this estimate depends on the ability of the modes in $\Phi$ to represent, individually or in combination, the actual lowest buckling mode of the original problem, where good accuracy can be achieved with reasonably selected approximation modes for $\boldsymbol{\Phi}$. 
To this extent, it is often possible to obtain an accurate estimate of the lowest buckling load using a single approximation mode, where $\Phi$ becomes a vector, and single-term stiffnesses are obtained from (21), leading to identical predictions to the Rayleigh quotient (Bažant \& Cedolin, 1991). In such a case, it is conceptually helpful to relate the geometric stiffness of the reduced problem directly to the energy absorbed $\left(\mathrm{k}_{\mathrm{G}}>0\right)$ or imparted $\left(\mathrm{k}_{\mathrm{G}}<0\right)$ by the equivalent rotational springs due to a unit $\mathrm{u}$, just as expressed by (10) for a SDOF system. Considering (17)-(19) with (21.c), this relationship is obtained as:

$$
\mathrm{k}_{\mathrm{G}}=\mathbf{t}_{\rho}^{\mathrm{T}} \operatorname{diag}\left(\mathbf{k}_{\rho}\right) \mathbf{t}_{\rho}=\sum_{\mathrm{i}=1}^{\mathrm{n}} \mathbf{k}_{\rho \mathrm{i}} \mathbf{t}_{\rho \mathrm{i}}^{2}
$$

in which $\mathbf{t}_{\rho}=$ transformation vector expressing the equivalent spring rotations in terms of $\mathrm{u}$ as follows:

$$
\rho=\mathbf{t}_{\rho} \mathrm{u}
$$

where:

$$
\mathbf{t}_{\rho}=\mathbf{T}_{\rho} \Phi
$$

Two examples are provided hereafter relating to buckling analysis of MDOF systems that consist of straight elements.

\section{Cantilever Truss with Flexible Lateral Restraints}

The cantilever truss shown in Figure 7 consists of five straight elements, and is laterally restrained with flexible springs at its two free nodes. The out-of-plane buckling of the truss is to be investigated under horizontal loading $(\mathrm{P})$, considering the interaction with a constant transverse loading $(\mathrm{Q})$. This problem resembles the lateral torsional buckling of cantilever beams subject to combined axial and bending actions, where in this case the buckling mode can be represented in terms of two displacement parameters $\mathbf{U}=\left\langle\mathbf{U}_{1} \mathbf{U}_{2}\right\rangle^{\mathrm{T}}$.

Assuming that the deformations up to the buckling point are negligible, matrix $\mathbf{T}_{\rho}$, relating $\boldsymbol{\rho}$ to $\mathbf{U}$ according to (18), and the element lengths are obtained as indicated in Figure 7. Assuming further that the diagonal and vertical members are relatively rigid, the axial forces 
are determined in terms of $\mathrm{P}$ and $\mathrm{Q}$ as shown in the figure, and hence the stiffnesses of the equivalent rotational springs ( $\mathbf{k}_{\rho}$ ) can be determined from (7). Of course, in the more general case, the axial forces for such an indeterminate structure may be determined from linear elastic planar analysis.

Considering first a zero transverse load $(\mathrm{Q}=0)$, the geometric stiffness is determined from (17) as:

$$
\mathbf{K}_{\mathrm{G}}=\frac{0.4167 \mathrm{P}}{\mathrm{L}}\left[\begin{array}{rr}
-1 & -1 \\
-1 & 1
\end{array}\right]
$$

while the corresponding material stiffness is simply given by:

$$
\mathbf{K}_{\mathrm{E}}=\left[\begin{array}{cc}
\mathrm{k} & 0 \\
0 & \mathrm{k}
\end{array}\right]
$$

The solution of the linear eigenvalue problem given by (4.a) leads to the following positive buckling load and associated mode:

$$
\begin{aligned}
& \operatorname{det}\left(\mathbf{K}_{\mathrm{T}}\right)=0 \Rightarrow \mathrm{P}_{\mathrm{c}}=1.697 \mathrm{~kL} \\
& \delta \mathbf{U}=\left\{\begin{array}{c}
1 \\
0.4142
\end{array}\right\}
\end{aligned}
$$

Before considering the influence of $\mathrm{Q}$ on the buckling load, it is useful to verify that a reduced problem with a single mode $\Phi$ identical to the exact buckling mode of (25.b) leads to the exact buckling load. In this case, the reduced stiffnesses are obtained as follows:

$$
\begin{aligned}
& \mathrm{k}_{\mathrm{E}}=\boldsymbol{\Phi}^{\mathrm{T}} \mathbf{K}_{\mathrm{E}} \boldsymbol{\Phi}=1.172 \mathrm{k} \\
& \mathrm{k}_{\mathrm{G}}=\sum_{\mathrm{i}=1}^{5} \mathbf{k}_{\rho \mathrm{\rho}} \mathbf{t}_{\rho \mathrm{i}}^{2} \equiv \boldsymbol{\Phi}^{\mathrm{T}} \mathbf{K}_{\mathrm{G}} \boldsymbol{\Phi}=-0.6904 \frac{\mathrm{P}}{\mathrm{L}}
\end{aligned}
$$

where,

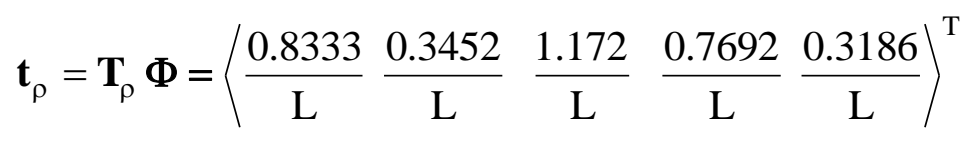


It is easily shown that the buckling load from the reduced SDOF problem, satisfying the condition $\left(\mathrm{k}_{\mathrm{T}}=0\right)$, is identical to the value determined in (25.a) for the original problem.

Now considering the effect of $\mathrm{Q}$ on the buckling load, it is of course straightforward to determine the new $\mathbf{K}_{\mathrm{G}}$ using the rotational spring analogy, and solving the resulting two degree-of-freedom eigenvalue problem, as shown later. However, the rotational spring analogy furnishes further conceptual power in this respect, where the influence of $\mathrm{Q}$ on $\mathrm{P}_{\mathrm{c}}$ may be estimated from the energy absorbed/imparted by the additional rotational springs corresponding to $\mathrm{Q}$ when the truss is subjected to the above mode:

$$
\mathrm{k}_{\mathrm{G}}^{\mathrm{Q}}=\sum_{\mathrm{i}=1}^{5} \mathbf{k}_{\rho \mathrm{i}}^{\mathrm{Q}} \mathbf{t}_{\rho \mathrm{i}}^{2} \equiv \boldsymbol{\Phi}^{\mathrm{T}} \mathbf{K}_{\mathrm{G}}^{\mathrm{Q}} \boldsymbol{\Phi}=-0.3431 \frac{\mathrm{Q}}{\mathrm{L}}
$$

Clearly, the influence of $(\mathrm{Q}>0)$ is destabilizing $\left(\mathrm{k}_{\mathrm{G}}^{\mathrm{Q}}<0\right)$, and leads to a smaller buckling load from the reduced problem, where the following value is obtained for $(\mathrm{Q}=0.2 \mathrm{~kL})$ :

$$
\mathrm{k}_{\mathrm{T}}=\mathrm{k}_{\mathrm{E}}+\mathrm{k}_{\mathrm{G}}=1.172 \mathrm{k}-0.6904 \frac{\mathrm{P}}{\mathrm{L}}-0.3431 \frac{\mathrm{Q}}{\mathrm{L}}=0 \Rightarrow \mathrm{P}_{\mathrm{c}}=1.598 \mathrm{~kL}
$$

Since the reduced SDOF problem is based on a mode which may no longer be true under combined $\mathrm{Q}$ and $\mathrm{P}$, the value of $\mathrm{P}_{c}$ obtained in (29) is an upper bound, and hence the effect of $(\mathrm{Q}=0.2 \mathrm{~kL})$ is to reduce the buckling load from $\left(\mathrm{P}_{\mathrm{c}}=1.697 \mathrm{~kL}\right)$ to $\left(\mathrm{P}_{\mathrm{c}} \leq 1.598 \mathrm{~kL}\right)$. This is now demonstrated by considering the original two degree-of-freedom problem under combined $\mathrm{Q}$ and $\mathrm{P}$, where the geometric stiffness matrix is obtained as:

$$
\mathbf{K}_{\mathrm{G}}=\frac{0.4167 \mathrm{P}}{\mathrm{L}}\left[\begin{array}{rr}
-1 & -1 \\
-1 & 1
\end{array}\right]+\frac{\mathrm{Q}}{\mathrm{L}}\left[\begin{array}{rr}
-1 & 1 \\
1 & -1
\end{array}\right]=\frac{0.4167 \mathrm{P}}{\mathrm{L}}\left[\begin{array}{rr}
-1 & -1 \\
-1 & 1
\end{array}\right]+0.2 \mathrm{k}\left[\begin{array}{rr}
-1 & 1 \\
1 & -1
\end{array}\right]
$$

leading to the following positive buckling load and associated mode:

$$
\begin{aligned}
& \operatorname{det}\left(\mathbf{K}_{\mathrm{T}}\right)=0 \Rightarrow \mathrm{P}_{\mathrm{c}}=1.576 \mathrm{~kL} \\
& \delta \mathbf{U}=\left\{\begin{array}{c}
1 \\
0.3136
\end{array}\right\}
\end{aligned}
$$


Clearly, the true buckling load in (31.a) for combined Q and P takes the estimated value in (29) as an upper limit, though the latter prediction still provides very good accuracy to within $1.4 \%$.

\section{Lateral Buckling of Frame}

The frame depicted in Figure 8 consists of four equal length elements, and is subjected to three vertical forces, determined by $\alpha_{i}$, such that the elements sustain identical compressive axial forces ( $-\mathrm{P})$ only. The lateral buckling of the frame is investigated using an assumed mode, obtained from a set of three lateral forces $\mathbf{Q}$ as given in Figure 8 , where $\mathrm{k}$ is a characteristic elastic lateral stiffness.

Ignoring the change of planar geometry due to loading, and assuming that the elements remain almost straight in the buckling mode, the lateral rotations $(\rho)$ of the four equivalent springs can be used to determine the geometric stiffness of the reduced SDOF problem:

$$
\mathrm{k}_{\mathrm{G}}=\sum_{\mathrm{i}=1}^{4} \mathbf{k}_{\rho \mathrm{i}} \mathbf{t}_{\rho \mathrm{i}}^{2}=\sum_{\mathrm{i}=1}^{4} \mathbf{k}_{\rho \mathrm{i}}\left(\frac{\boldsymbol{\rho}_{\mathrm{i}}}{\mathrm{u}}\right)^{2}=-\frac{0.625 \mathrm{P}}{\mathrm{L}}
$$

On the other hand, the material stiffness of the reduced problem, defined in (21.b), is conveniently obtained from the work done by the normalized lateral forces as follows:

$$
\mathrm{k}_{\mathrm{E}}=\boldsymbol{\Phi}^{\mathrm{T}} \mathbf{K}_{\mathrm{E}} \boldsymbol{\Phi}=\frac{1}{\mathrm{u}^{2}} \mathbf{U}^{\mathrm{T}} \mathbf{K}_{\mathrm{E}} \mathbf{U}=\frac{1}{\mathrm{u}^{2}} \mathbf{U}^{\mathrm{T}} \mathbf{Q}=\mathrm{k}
$$

Therefore, buckling for the above assumed lateral mode occurs at the following load:

$$
\mathrm{k}_{\mathrm{T}}=\mathrm{k}_{\mathrm{E}}+\mathrm{k}_{\mathrm{G}}=0 \Rightarrow \mathrm{P}_{\mathrm{c}}=1.6 \mathrm{~kL}
$$

Of course, the buckling load may be overestimated by the above assumed mode, though an improved approximation could be obtained by considering various realistic modes, individually or in combination. Additionally, the assumption of straight elements neglects the local bending deformations in the buckling mode, thus ignoring the local equivalent rotational springs associated with the $\mathrm{P}-\delta$ effect, as discussed in the previous section. Since the ignored local equivalent springs for this problem would have negative rotational stiffness, 
with all the axial forces being negative, this leads to the exclusion of a destabilizing portion of the geometric stiffness and, hence, to a further overestimate of the buckling load. However, this relatively small inaccuracy for this problem can be easily reduced by increasing the number of straight elements, as noted in the previous section, or by accounting for local geometric nonlinearity, as elaborated in the first example of the following section.

\section{CONTINUOUS SYSTEMS}

When the trajectory of an axial force due to a buckling mode continuously varies over the structural domain, the geometric stiffness can be obtained as the integration of contributions from distributed equivalent rotational springs. In the most general case, the geometric stiffness may be due to both discrete and distributed equivalent rotational springs, and therefore a general expression including both types of equivalent springs is required. Assuming that the buckling mode can be represented by a discrete number of parameters $\mathbf{U}$ through pre-defined shape functions, the geometric stiffness matrix can be determined from:

$$
\mathbf{K}_{\mathrm{G}}=\mathbf{T}_{\rho}^{\mathrm{T}} \operatorname{diag}\left(\mathbf{k}_{\rho}\right) \mathbf{T}_{\rho}+\int \mathbf{T}_{\theta}^{\mathrm{T}} \mathrm{k}_{\theta} \mathbf{T}_{\theta} \mathrm{dx}
$$

Clearly, the first part of $\mathbf{K}_{\mathrm{G}}$ in (35) deals with the discrete equivalent rotational springs in the same way as for MDOF systems, as expressed by (17). Furthermore, the second part is due to the distributed equivalent rotational springs, where, as given by (8), $\mathrm{k}_{\theta}$ is identical to the axial force at the corresponding location, and $\mathbf{T}_{\theta}$ is a row vector of domain functions expressing a first-order kinematic relationship between the rotations of the equivalent springs and $\mathbf{U}$ :

$$
\theta=\mathbf{T}_{\theta} \mathbf{U}
$$

The above expression for $\mathbf{K}_{\mathrm{G}}$ can be generalized further to operate on the level of principal stresses, instead of resultant axial forces. This enables the application of the rotational spring analogy to a wide range of geometrically nonlinear problems, including the lateral torsional buckling of beams and the buckling of plates, as shown later in several examples. 
On the other hand, the material stiffness matrix $\left(\mathbf{K}_{\mathrm{E}}\right)$ can be determined from standard discretization techniques, such as ones based on linear finite elements, considering the same displacement parameters $\mathbf{U}$.

Finally, the accuracy of the buckling load prediction depends on the ability of the assumed shape functions to represent the lowest buckling mode. Provided all sources of geometric nonlinearity are accounted for by means of discrete and/or distributed equivalent rotational springs, the predicted buckling load will be an upper bound on the actual value, just as with problem reduction for MDOF discrete systems using approximation modes.

\section{Cantilever Column with Flexible Support}

The cantilever column shown in Figure 9 has a flexible rotational support, where the support stiffness is related to the column flexural stiffness using a non-dimensional parameter $(\alpha)$. The column is discretized using a bending element accounting for local geometric nonlinearity, where a two degree-of-freedom buckling problem is considered, with $\mathbf{U}_{1}$ referring to the element chord rotation, and $\mathbf{U}_{2}$ used to approximate the local element deformations $\mathrm{v}(\mathrm{x})$ by means of the cubic shape function in Figure 9. According to this discretization, and neglecting the change in geometry up to buckling, the geometric stiffness matrix is obtained from (35) as:

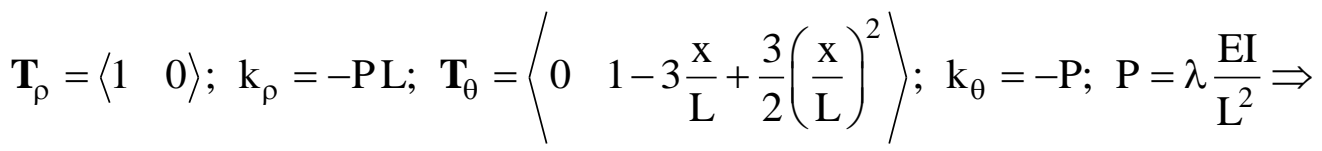

$$
\begin{aligned}
& \mathbf{K}_{\mathrm{G}}=-\frac{\lambda \mathrm{EI}}{\mathrm{L}}\left[\begin{array}{cc}
1 & 0 \\
0 & 0.2
\end{array}\right] \quad \text { (with local geometric nonlinearity) } \\
& \mathbf{K}_{\mathrm{G}}=-\frac{\lambda \mathrm{EI}}{\mathrm{L}}\left[\begin{array}{ll}
1 & 0 \\
0 & 0
\end{array}\right] \quad \text { (without local geometric nonlinearity) }
\end{aligned}
$$

In the above, and for comparison purposes, the geometric stiffness is determined with and without local geometric nonlinearity, where in the latter case the contribution of the distributed equivalent rotational springs in (35) is ignored. 
On the other hand, the material stiffness is obtained for the above discretization using standard linear analysis techniques employing the principle of virtual work:

$$
\begin{aligned}
& \theta_{\mathrm{s}}=\text { support spring rotation }=\mathbf{T} \mathbf{U}, \quad \mathbf{T}=\left\langle\begin{array}{ll}
1 & 1
\end{array}\right\rangle \\
& \left.\kappa=\text { column curvature }=\frac{\mathrm{d} \theta}{\mathrm{dx}}=\mathbf{B} \mathbf{U}, \quad \mathbf{B}=\left\langle\begin{array}{ll}
0 & \frac{-3}{\mathrm{~L}}\left(1-\frac{\mathrm{x}}{\mathrm{L}}\right)
\end{array}\right)\right\rangle \Rightarrow \\
& \mathbf{K}_{\mathrm{E}}=\mathbf{T}^{\mathrm{T}} \mathrm{k} \mathbf{T}+\int_{0}^{\mathrm{L}} \mathbf{B}^{\mathrm{T}} \mathrm{EI} \mathbf{B} \mathrm{dx}=\frac{\mathrm{EI}}{\mathrm{L}}\left[\begin{array}{cc}
\alpha & \alpha \\
\alpha & \alpha+3
\end{array}\right]
\end{aligned}
$$

The lowest buckling load is obtained from the singularity of $\mathbf{K}_{\mathrm{T}}$, as required by (4.a), leading to:

$$
\begin{array}{ll}
\lambda_{c}=\frac{5 \alpha}{\alpha+2.5+\sqrt{(\alpha+2.5)^{2}-\frac{5 \alpha}{3}}} & \text { (with local geometric nonlinearity) } \\
\lambda_{c}=\frac{3 \alpha}{\alpha+3} & \quad \text { (without local geometric nonlinearity) }
\end{array}
$$

The exact buckling load, on the other hand, can be easily established from equilibrium in the perturbed configuration, where the critical load factor is the root of the following equation:

$$
\sqrt{\lambda_{\mathrm{c}}} \tan \left(\sqrt{\lambda_{\mathrm{c}}}\right)-\alpha=0 \quad \text { (exact) }
$$

The two buckling loads factors predicted using the rotational spring analogy, with/without local nonlinearity, are compared in Figure 10 against the exact value for a range of rotational support stiffnesses as determined by the non-dimensional $\alpha$ parameter. Clearly, the exact solution is bounded by $\left(\lambda_{c}=\alpha\right)$ for a relatively flexible support and by $\left(\lambda_{c}=\pi^{2} / 4 \approx 2.467\right)$ for a relatively rigid support, as expected for this problem. Furthermore, the proposed rotational spring analogy provides excellent comparison over the full range of $\alpha$ when accounting for local geometric nonlinearity. Despite the approximation of $\mathrm{v}(\mathrm{x})$ using a simple cubic function, the expected overestimation of the critical load factor is limited to a maximum of $1.3 \%$, which occurs at the rigid support limit $(\alpha \rightarrow \infty)$ where $\left(\lambda_{c} \rightarrow 2.5\right)$. However, for the case ignoring local geometric nonlinearity, the comparison is good for small $\alpha$, but deteriorates to a maximum overestimation of $21.6 \%$ in the rigid limit where $\left(\lambda_{c} \rightarrow 3\right)$. 
Observing that the ratio $\left(\delta \mathbf{U}_{2} / \delta \mathbf{U}_{1}\right)$ in the buckling mode is obtained as $\left(\lambda_{\mathrm{c}} / \alpha-1\right)$, the local buckling deformations represented by $\delta \mathbf{U}_{2}$ are relatively small for small $\alpha$, where $\left(\lambda_{\mathrm{c}} \approx \alpha\right)$, and hence the influence of local geometric nonlinearity becomes also small. However, $\delta \mathbf{U}_{2}$ becomes comparable to $-\delta \mathbf{U}_{1}$ as the support stiffness is increased to the rigid limit, leading to considerable further overestimation of the critical load when local nonlinearity is ignored, since the excluded local equivalent springs are all destabilizing $\left(\mathbf{k}_{\theta}<0\right)$. Of course, whether local geometric nonlinearity is included or excluded, convergence to the exact solution is guaranteed in the limit as more elements are used.

\section{Arch Subject to Distributed Load}

Consider a circular arch with radius $\mathrm{R}$ and subtended angle $2 \alpha$, subject to a distributed load (w) that is initially normal to the arch, as shown in Figure 11. The buckling of this structure under w which remains normal to the arch as it deflects, similar to the action of hydrostatic pressure, was investigated previously using equilibrium in the perturbed configuration (Timoshenko \& Gere, 1961; Allen \& Bulson, 1980). In addition to this loading case, consideration is given here using the proposed rotational spring analogy to loading which maintains a fixed direction as in the initial configuration, and to radial loading which always points to the centre of the arch.

Ignoring the arch deflections up to buckling, a single parameter $\mathrm{U}$ is used to represent the buckling mode, expressed in terms of shape functions for the tangential and radial displacements:

$$
\begin{aligned}
& \mathrm{u}(\mathrm{x})=\frac{\mathrm{U} \alpha}{\pi}\left[\cos \left(\frac{\pi \mathrm{x}}{\alpha \mathrm{R}}\right)+1\right] \\
& \mathrm{v}(\mathrm{x})=\mathrm{U} \sin \left(\frac{\pi \mathrm{x}}{\alpha \mathrm{R}}\right)
\end{aligned}
$$

where $\mathrm{x}=$ tangential coordinate along the arch (Figure 11).

The above mode corresponds to the assumption of an axially inextensible arch, defined in terms of a zero centroidal axial strain: 


$$
\varepsilon_{\mathrm{c}}=\mathrm{u}^{\prime}+\mathrm{v} / \mathrm{R}=0
$$

The remaining generalized strain for the arch is the curvature, which is given to a first-order in terms of $\mathrm{U}$ as follows:

$$
\kappa=\mathrm{v}^{\prime \prime}+\mathrm{v} / \mathrm{R}^{2}=\frac{\mathrm{U}}{\mathrm{R}^{2}}\left(1-\frac{\pi^{2}}{\alpha^{2}}\right) \sin \left(\frac{\pi \mathrm{x}}{\alpha \mathrm{R}}\right)
$$

leading to the following material stiffness:

$$
\mathrm{K}_{\mathrm{E}}=\int_{-\alpha \mathrm{R}}^{\alpha \mathrm{R}} \mathrm{EI}\left(\frac{\kappa}{\mathrm{U}}\right)^{2} \mathrm{dx}=\frac{\alpha \mathrm{EI}}{\mathrm{R}^{3}}\left(\frac{\pi^{2}}{\alpha^{2}}-1\right)^{2}
$$

Considering first the load with fixed direction, the geometric stiffness can be determined from distributed equivalent rotational springs corresponding to the arch axial force, as illustrated in Figure 11, which respond to the tangential arch rotation:

$$
\begin{aligned}
& \mathrm{k}_{\theta_{\mathrm{t}}}=\mathrm{F}=-\mathrm{w} \mathrm{R}=\text { distributed tangential stiffness per unit arch length } \\
& \theta_{\mathrm{t}}=\text { tangential rotation }=\mathrm{v}^{\prime}-\frac{\mathrm{u}}{\mathrm{R}}=\frac{\mathrm{U} \alpha}{\pi \mathrm{R}}\left[\left(\frac{\pi^{2}}{\alpha^{2}}-1\right) \cos \left(\frac{\pi \mathrm{x}}{\alpha \mathrm{R}}\right)-1\right] \\
& \mathrm{K}_{\mathrm{G}}=\int_{-\alpha \mathrm{R}}^{\alpha \mathrm{R}} \mathrm{k}_{\theta_{\mathrm{t}}}\left(\frac{\theta_{\mathrm{t}}}{\mathrm{U}}\right)^{2} \mathrm{dx}=-\frac{\mathrm{w} \alpha^{3}}{\pi^{2}}\left[2+\left(\frac{\pi^{2}}{\alpha^{2}}-1\right)^{2}\right] \Rightarrow
\end{aligned}
$$

Therefore, the buckling load satisfying the condition of zero $\mathrm{K}_{\mathrm{T}}$ is obtained using (46) and (48) as:

$$
\mathrm{w}_{\mathrm{c}}=\frac{\pi^{2} \mathrm{EI}}{\alpha^{2} \mathrm{R}^{3}} /\left[2 /\left(\frac{\pi^{2}}{\alpha^{2}}-1\right)^{2}+1\right] \quad \text { (fixed load direction) }
$$

Considering next the load with radial direction, the geometric stiffness is due to the axial force in the arch, as given before by (48), in addition to the radial loading. This additional contribution can be obtained from distributed equivalent radial springs, which have a stabilizing rotational stiffness as discussed previously, leading to the following overall geometric stiffness: 


$$
\left.\begin{array}{l}
\mathrm{k}_{\theta_{\mathrm{r}}}=\mathrm{w} \mathrm{R}=\text { distributed radial stiffness per unit arch length } \\
\theta_{\mathrm{r}}=\operatorname{radial} \text { rotation }=-\frac{\mathrm{u}}{\mathrm{R}}=-\frac{\mathrm{U} \alpha}{\pi \mathrm{R}}\left[\cos \left(\frac{\pi \mathrm{x}}{\alpha \mathrm{R}}\right)+1\right]
\end{array}\right\} \Rightarrow
$$

Therefore, the buckling load is obtained using (46) and (51) as:

$$
\mathrm{w}_{\mathrm{c}}=\frac{\mathrm{EI}}{\mathrm{R}^{3}}\left(\frac{\pi^{2}}{\alpha^{2}}-1\right)^{2} /\left(\frac{\pi^{2}}{\alpha^{2}}-2\right) \quad(\text { radial load direction })
$$

Finally, consideration is given to loading which maintains a normal direction to the deformed arch configuration, similar to the action of hydrostatic pressure. Such loading is often nonconservative, but can be shown to be conservative when applied uniformly to closed line boundaries or to open line boundaries with fixed ends (Hodges, 1999), as is the case for this arch problem. However, unlike radial loads, the geometric stiffness associated with normal loads cannot be represented by equivalent rotational (or translational) springs, since it is manifested in terms of a coupling between the tangential rotation and the axial component of the load. This additional contribution can be obtained according to (5.c) from the secondorder variation of the work done by the total loads $(\lambda W)$ with respect to $\mathrm{U}$, evaluated at the initial configuration ( $U=0$ ). For constant normal loading, the work done is directly related to the aggregate area enclosed between the initial and deformed trajectories, which for the inextensible arch is expressed to a second-order as:

$$
\lambda W=\mathrm{w} \int_{-\alpha \mathrm{R}}^{\alpha \mathrm{R}} \mathrm{dA}=\mathrm{w} \int_{-\alpha \mathrm{R}}^{\alpha \mathrm{R}}\left[\frac{-\mathrm{v}+\sin \left(\theta_{\mathrm{t}}\right) \mathrm{u}-\cos \left(\theta_{\mathrm{t}}\right) \mathrm{v}}{2}\right] \mathrm{dx}=\mathrm{w} \int_{-\alpha \mathrm{R}}^{\alpha \mathrm{R}}\left[-\mathrm{v}+\frac{\theta_{\mathrm{t}} \mathrm{u}}{2}+\cdots\right] \mathrm{dx}
$$

leading to the additional geometric stiffness contribution:

$$
-\lambda \frac{\partial^{2} W}{\partial U^{2}}=-w \int_{-\alpha R}^{\alpha R}\left(\frac{\theta_{t}}{U}\right)\left(\frac{u}{U}\right) d x
$$


Therefore, the overall geometric stiffness, due to the axial arch force and the normal load, involves the following modification to (48):

$$
\mathrm{K}_{\mathrm{G}}=\int_{-\alpha \mathrm{R}}^{\alpha \mathrm{R}}\left[\mathrm{k}_{\theta_{\mathrm{t}}}\left(\frac{\theta_{\mathrm{t}}}{\mathrm{U}}\right)^{2}-\mathrm{w}\left(\frac{\theta_{\mathrm{t}}}{\mathrm{U}}\right)\left(\frac{\mathrm{u}}{\mathrm{U}}\right)\right] \mathrm{dx}=-\mathrm{w} \alpha\left(\frac{\pi^{2}}{\alpha^{2}}-1\right)
$$

which in combination with (46) leads to the corresponding buckling load:

$$
\mathrm{w}_{\mathrm{c}}=\frac{\mathrm{EI}}{\mathrm{R}^{3}}\left(\frac{\pi^{2}}{\alpha^{2}}-1\right) \quad \text { (normal load direction) }
$$

Importantly, the buckling load in (56) is identical to previous predictions for the normal loading case (Timoshenko \& Gere, 1961; Allen and Bulson, 1980). A comparison between the buckling loads for the three cases considered above is shown in Figure 12 for a range of subtended arch angles, where it is clear over this range that the normal and radial loading are consistently associated with the smallest and largest buckling loads, respectively. It should be noted that the above buckling load predictions are based on a single assumed mode, and hence are upper bounds on the respective exact values. Nevertheless, convergence studies using high-order polynomial approximation (up to $11^{\text {th }}$ and $12^{\text {th }}$ order for $\mathrm{u}(\mathrm{x})$ and $\mathrm{v}(\mathrm{x})$, respectively) have shown that the assumed mode is exact for radial/normal loads and almost exact for fixed loads, where the maximum error in the buckling load is within $0.05 \%$ for the range of $\alpha$ considered.

\section{Column Subject to Torsional Buckling}

In the previous examples, the geometric stiffness is established for axial members with constant cross-sectional stresses, such that all stresses over a specific cross-section are subject to the same lateral buckling rotation, thus enabling the use of a single equivalent rotational spring for the whole cross-section. However, when the buckling of an axial member involves a torsional mode, different stress points over the cross-section could be subject to different lateral rotations, offering different contributions to the overall cross-sectional geometric 
stiffness. In this case, the cross-sectional geometric stiffness is obtained from the integrated contributions of equivalent rotational springs distributed over the cross-section.

To demonstrate the above point, consider the column cross-section in Figure 13, which is subject to a varying twist rotation $(\omega)$ over the $\mathrm{x}$-axis, typically passing through the shear centre. The influence of geometric nonlinearity can be represented by equivalent rotational springs distributed over both the column length and cross-sectional area, where:

$$
\begin{aligned}
& \mathrm{k}_{\theta}=\sigma_{\mathrm{x}}=-\frac{\mathrm{P}}{\mathrm{A}}=\text { distributed rotational stiffness per unit length and unit area } \\
& \theta=\sqrt{\mathrm{y}^{2}+\mathrm{z}^{2}} \frac{\mathrm{d} \omega}{\mathrm{dx}}=\text { lateral rotation of equivalent springs }
\end{aligned}
$$

If $\omega$ is approximated over the column length in terms of parameters $\mathbf{U}$ such that:

$$
\frac{\mathrm{d} \omega}{\mathrm{dx}}=\mathbf{T}_{\omega} \mathbf{U}
$$

then in view of (36) and (59):

$$
\mathbf{T}_{\theta}=\sqrt{\mathrm{y}^{2}+\mathrm{z}^{2}} \mathbf{T}_{\omega}
$$

Now, generalizing (35) the geometric stiffness matrix is obtained as:

$$
\mathbf{K}_{\mathrm{G}}=\iint \mathbf{T}_{\theta}^{\mathrm{T}} \mathrm{k}_{\theta} \mathbf{T}_{\theta} \mathrm{dAdx}=\int \mathbf{T}_{\omega}^{\mathrm{T}} \mathbf{T}_{\omega}\left(\int \sigma_{\mathrm{x}}\left(\mathrm{y}^{2}+\mathrm{z}^{2}\right) \mathrm{dA}\right) \mathrm{dx}=-\int \frac{\mathrm{PI}_{0}}{\mathrm{~A}} \mathbf{T}_{\omega}^{\mathrm{T}} \mathbf{T}_{\omega} \mathrm{dx}
$$

where $\mathrm{I}_{0}=$ polar second moment of area.

Considering a cantilever column where the torsional mode is approximated by a single function:

$$
\omega(\mathrm{x})=\mathrm{U} \frac{\mathrm{x}}{\mathrm{L}} \Rightarrow \mathrm{T}_{\omega}=\frac{1}{\mathrm{U}} \frac{\mathrm{d} \omega}{\mathrm{dx}}=\frac{1}{\mathrm{~L}}
$$

the following geometric stiffness is obtained:

$$
\mathrm{K}_{\mathrm{G}}=-\int \frac{\mathrm{PI}_{0}}{\mathrm{~A}}\left(\mathrm{~T}_{\omega}\right)^{2} \mathrm{dx}=-\frac{\mathrm{PI}_{0}}{\mathrm{AL}}
$$

Neglecting warping deformations, the material stiffness is simply determined from: 


$$
\mathrm{K}_{\mathrm{E}}=\int \mathrm{GJ} \mathbf{T}_{\omega}^{\mathrm{T}} \mathbf{T}_{\omega} \mathrm{dx}=\frac{\mathrm{GJ}}{\mathrm{L}}
$$

where $\mathrm{GJ}=$ torsional rigidity.

Therefore, considering (63) and (64), buckling occurs at:

$$
\mathrm{P}_{\mathrm{c}}=\frac{\mathrm{GJ} \mathrm{A}}{\mathrm{I}_{0}}
$$

which is identical to the theoretical value (Timoshenko \& Gere, 1961).

\section{Beam Subject to Lateral-Torsional Buckling}

Taking the last generalization of the rotational spring analogy further, consideration is given here to a bending problem where the geometric stiffness arises from direct and shear stresses that vary over the member cross-section. The lateral torsional buckling of a simply supported beam with lateral and torsional end restraints is investigated under a midspan load $(\mathrm{P})$, applied at a distance (a) above the centroidal axis. The beam has a thin rectangular section, and is subject to bending about its major axis, leading to the typical linear and quadratic distributions for direct $\left(\sigma_{\mathrm{x}}\right)$ and shear $\left(\tau_{\mathrm{xz}}\right)$ stresses, respectively, as illustrated in Figure 14 .

Distributed equivalent rotational springs should be associated with the principal stresses at specific points over the cross-section, though considerable simplification is achieved if one set of distributed springs is used for $\sigma_{\mathrm{x}}$, and two sets of such springs are used for the principal stresses corresponding to $\tau_{\mathrm{xz}}$ (Figure 14). Therefore, the stiffnesses of the three distributed equivalent springs and their lateral rotations due to lateral deflection (v) and twist rotation $(\omega)$, neglecting the initial deformations due to $\mathrm{P}$, are given by:

$$
\mathbf{k}_{\theta}=\left\{\begin{array}{c}
\sigma_{\mathrm{x}} \\
\tau_{\mathrm{xz}} \\
-\tau_{\mathrm{xz}}
\end{array}\right\} ; \quad \boldsymbol{\theta}=\mathbf{Z}\left\{\begin{array}{c}
\omega \\
\mathrm{d} \omega / \mathrm{dx} \\
\mathrm{dv} / \mathrm{dx}
\end{array}\right\} ; \quad \mathbf{Z}=\left[\begin{array}{ccc}
0 & -\mathrm{z} & 1 \\
1 / \sqrt{2} & \mathrm{z} / \sqrt{2} & -1 / \sqrt{2} \\
1 / \sqrt{2} & -\mathrm{z} / \sqrt{2} & 1 / \sqrt{2}
\end{array}\right]
$$

If $\mathrm{v}$ and $\omega$ are approximated in terms of parameters $\mathbf{U}, \mathbf{T}_{\theta}$ of (36) becomes a matrix obtained from: 


$$
\left\{\begin{array}{c}
\omega \\
\mathrm{d} \omega / \mathrm{dx} \\
\mathrm{dv} / \mathrm{dx}
\end{array}\right\}=\mathbf{T}_{\omega \mathrm{v}} \mathbf{U} ; \quad \mathbf{T}_{\theta}=\mathbf{Z} \mathbf{T}_{\omega \mathrm{v}}
$$

and the geometric stiffness matrix arising from the internal stresses is determined as:

$$
\begin{aligned}
& \mathbf{K}_{\mathrm{G}}=\iint \mathbf{T}_{\theta}^{\mathrm{T}} \operatorname{diag}\left(\mathbf{k}_{\theta}\right) \mathbf{T}_{\theta} \mathrm{dAdx}=\int \mathbf{T}_{\omega \mathrm{v}}^{\mathrm{T}}\left(\int \mathbf{Z}^{\mathrm{T}} \operatorname{diag}\left(\mathbf{k}_{\theta}\right) \mathbf{Z d A}\right) \mathbf{T}_{\omega \mathrm{v}} \mathrm{dx} \Rightarrow \\
& \mathbf{K}_{\mathrm{G}}=\int \mathbf{T}_{\omega \mathrm{v}}^{\mathrm{T}}\left[\begin{array}{ccc}
0 & 0 & \mathrm{Q} \\
0 & 0 & \mathrm{M} \\
\mathrm{Q} & \mathrm{M} & 0
\end{array}\right] \mathbf{T}_{\omega \mathrm{v}} \mathrm{dx}
\end{aligned}
$$

It is important to note that the above result is only applicable to doubly symmetric sections subject to bending moment $(\mathrm{M})$ and shear force $(\mathrm{Q})$, which are defined as:

$$
M=\int-\sigma_{x} z d A ; \quad Q=\int-\tau_{x z} d A=d M / d x
$$

though the rotational spring analogy may be generalized further to other cross-sectional shapes and stress distributions.

Assuming that the lateral buckling mode is defined in terms of two parameters as follows:

$$
\omega(\mathrm{x})=\mathbf{U}_{1} \sin (\pi \mathrm{x} / \mathrm{L}) ; \quad \mathrm{v}(\mathrm{x})=\mathbf{U}_{2} \sin (\pi \mathrm{x} / \mathrm{L})
$$

the transformation matrix needed in (69) is obtained as:

$$
\mathbf{T}_{\omega \mathrm{v}}=\left[\begin{array}{cc}
\sin (\pi \mathrm{x} / \mathrm{L}) & 0 \\
(\pi / \mathrm{L}) \cos (\pi \mathrm{x} / \mathrm{L}) & 0 \\
0 & (\pi / \mathrm{L}) \cos (\pi \mathrm{x} / \mathrm{L})
\end{array}\right]
$$

Of course, there is an additional contribution to $\mathbf{K}_{\mathrm{G}}$ due to $\mathrm{P}$ applied above the centroidal axis, which can be represented by a discrete equivalent rotational spring:

$$
\mathrm{k}_{\rho}=-\mathrm{Pa} ; \quad \mathbf{T}_{\rho}=\left\langle\frac{\omega(\mathrm{L} / 2)}{\mathbf{U}_{1}} \quad 0\right\rangle=\left\langle\begin{array}{ll}
1 & 0
\end{array}\right\rangle
$$

and which is clearly destabilizing for $(\mathrm{Pa}>0)$.

Therefore, with the bending moment and shear force varying as: 


$$
M=\left\{\begin{array}{cc}
P x / 2 & x \leq L / 2 \\
P(L-x) / 2 & x \geq L / 2
\end{array} ; Q=\left\{\begin{array}{cc}
P / 2 & x<L / 2 \\
-P / 2 & x>L / 2
\end{array}\right.\right.
$$

the overall geometric stiffness matrix is obtained in terms of P:

$$
\mathbf{K}_{\mathrm{G}}=\mathbf{T}_{\rho}^{\mathrm{T}} \mathrm{k}_{\rho} \mathbf{T}_{\rho}+\int_{0}^{\mathrm{L}} \mathbf{T}_{\omega \mathrm{v}}^{\mathrm{T}}\left[\begin{array}{ccc}
0 & 0 & \mathrm{Q} \\
0 & 0 & \mathrm{M} \\
\mathrm{Q} & \mathrm{M} & 0
\end{array}\right] \mathbf{T}_{\omega \mathrm{v}} \mathrm{dx}=\mathrm{P}\left[\begin{array}{cc}
-\mathrm{a} & \frac{\pi^{2}+4}{16} \\
\frac{\pi^{2}+4}{16} & 0
\end{array}\right]
$$

On the other hand the material stiffness is determined from the assumed variation of twist $(\psi)$ and curvature $(\kappa)$ generalized strains:

$$
\left\{\begin{array}{l}
\psi \\
\kappa
\end{array}\right\}=\left\{\begin{array}{c}
\mathrm{d} \omega / \mathrm{dx} \\
\mathrm{d}^{2} \mathrm{v} / \mathrm{dx}^{2}
\end{array}\right\}=\mathbf{B} \mathbf{U} ; \quad \mathbf{B}=\left[\begin{array}{cc}
(\pi \mathrm{x} / \mathrm{L}) \cos (\pi \mathrm{x} / \mathrm{L}) & 0 \\
0 & -(\pi \mathrm{x} / \mathrm{L})^{2} \sin (\pi \mathrm{x} / \mathrm{L})
\end{array}\right]
$$

leading to:

$$
\mathbf{K}_{\mathrm{E}}=\int_{0}^{\mathrm{L}} \mathbf{B}^{\mathrm{T}}\left[\begin{array}{cc}
\mathrm{GJ} & 0 \\
0 & \mathrm{EI}
\end{array}\right] \mathbf{B} \mathrm{dx}=\left[\begin{array}{cc}
\frac{\pi^{2} \mathrm{GJ}}{2 \mathrm{~L}} & 0 \\
0 & \frac{\pi^{4} \mathrm{EI}}{2 \mathrm{~L}^{3}}
\end{array}\right]
$$

Therefore, the lowest buckling load is obtained from the singularity of $\mathbf{K}_{\mathrm{T}}$ as:

$$
\mathrm{P}_{\mathrm{c}}=\frac{17.88}{\mathrm{~L}^{2}}\left[-1.812 \frac{\mathrm{EIa}}{\mathrm{L}}+\sqrt{\mathrm{EIGJ}+\left(1.812 \frac{\mathrm{EIa}}{\mathrm{L}}\right)^{2}}\right]
$$

which compares favorably to previous theoretical predictions (Timoshenko \& Gere, 1961) to within $6 \%$. Of course, this discrepancy is due to the assumed mode in (71), where convergence to the theoretical solution is guaranteed with improved approximation of the buckling mode.

\section{Plate Buckling}

As a final further generalization of the rotational spring analogy, consideration is given here to a two-dimensional plate buckling problem. For this purpose, a simply supported thin rectangular plate, with coordinates $(0 \leq \mathrm{x} \leq \mathrm{a})$ and $(0 \leq \mathrm{y} \leq \mathrm{b})$, is investigated under the 
combined compressive action of uniformly distributed edge forces, $\mathrm{N}_{\mathrm{y}}$ and $\mathrm{N}_{\mathrm{x}}$. In this case, the geometric stiffness is obtained from two sets of equivalent rotational springs distributed over the plate area, with rotations equal to the slopes of the lateral buckling mode (w):

$$
\mathbf{k}_{\theta}=\left\{\begin{array}{l}
-\mathrm{N}_{\mathrm{x}} \\
-\mathrm{N}_{\mathrm{y}}
\end{array}\right\} ; \quad \boldsymbol{\theta}=\left\{\begin{array}{l}
\partial \mathrm{w} / \partial \mathrm{x} \\
\partial \mathrm{w} / \partial \mathrm{y}
\end{array}\right\}=\mathbf{T}_{\theta} \mathbf{U}
$$

Assuming a single mode, satisfying the support boundary conditions, with the following general form:

$$
\mathrm{w}(\mathrm{x}, \mathrm{y})=\mathrm{U} \sin \left(\frac{\mathrm{m} \pi}{\mathrm{a}} \mathrm{x}\right) \sin \left(\frac{\mathrm{n} \pi}{\mathrm{b}} \mathrm{y}\right)(\mathrm{m}, \mathrm{n}>1) ; \quad \mathbf{T}_{\theta}=\left\{\begin{array}{l}
\frac{\mathrm{m} \pi}{\mathrm{a}} \cos \left(\frac{\mathrm{m} \pi}{\mathrm{a}} \mathrm{x}\right) \sin \left(\frac{\mathrm{n} \pi}{\mathrm{b}} \mathrm{y}\right) \\
\frac{\mathrm{n} \pi}{\mathrm{b}} \sin \left(\frac{\mathrm{m} \pi}{\mathrm{a}} \mathrm{x}\right) \cos \left(\frac{\mathrm{n} \pi}{\mathrm{b}} \mathrm{y}\right)
\end{array}\right\}
$$

the corresponding geometric stiffness is obtained as:

$$
\mathrm{K}_{\mathrm{G}}=\int \mathbf{T}_{\theta}^{\mathrm{T}} \operatorname{diag}\left(\mathbf{k}_{\theta}\right) \mathbf{T}_{\theta} \mathrm{dA}=-\frac{\pi^{2} \mathrm{ab}}{4}\left(\mathrm{~N}_{\mathrm{x}} \frac{\mathrm{m}^{2}}{\mathrm{a}^{2}}+\mathrm{N}_{\mathrm{y}} \frac{\mathrm{n}^{2}}{\mathrm{~b}^{2}}\right)
$$

On the other hand, the material stiffness can be determined using standard discretization principles. Assuming linear elastic isotropic material the following value is obtained:

$$
K_{E}=\int \mathbf{B}^{\mathrm{T}} \mathbf{D B} \mathrm{BA}=\frac{\mathrm{Et}^{3}}{12\left(1-v^{2}\right)} \frac{\pi^{4} \mathrm{ab}}{4}\left(\frac{\mathrm{m}^{2}}{\mathrm{a}^{2}}+\frac{\mathrm{n}^{2}}{\mathrm{~b}^{2}}\right)^{2}
$$

where $\mathrm{E}=$ Young's modulus, $v=$ Poisson's ratio, and $\mathrm{t}=$ plate thickness.

Therefore, taking $\mathrm{r}$ to be the ratio $\left(\mathrm{N}_{\mathrm{y}} / \mathrm{N}_{\mathrm{x}}\right)$, the buckling load corresponding to the assumed mode is determined as:

$$
\mathrm{N}_{\mathrm{xc}}=\frac{\pi^{2} E \mathrm{t}^{3}}{12\left(1-v^{2}\right)}\left(\frac{\mathrm{m}^{2}}{\mathrm{a}^{2}}+\frac{\mathrm{n}^{2}}{\mathrm{~b}^{2}}\right)^{2} /\left(\frac{\mathrm{m}^{2}}{\mathrm{a}^{2}}+\mathrm{r} \frac{\mathrm{n}^{2}}{\mathrm{~b}^{2}}\right)
$$

which is identical to previous theoretical predictions (e.g. Allen \& Bulson, 1980). 


\section{CONCLUSION}

A rotational spring analogy is proposed for formulating the geometric stiffness of nonlinear structural systems, which can be employed within an intuitive framework based on linear analysis principles. According to this analogy, the geometric stiffness matrix can be determined from discrete and/or distributed equivalent rotational springs, which represent contributions from internal axial forces and/or principal stresses as well as from some types of externally applied conservative loads.

The rotational spring analogy is initially formulated to deal with the deflecting trajectory of the axial force in axially-loaded members and with the changing direction of conservative radial loads. For axial members that remain almost straight and for radial point loads, the analogy utilizes discrete equivalent rotational springs, which deform upon lateral rotation of the axial-member/radial-load, with a spring stiffness as the product of the axial-force/radialload and the member-length/radial-distance. The analogy is easily generalized to deal with varying axial force, distributed radial load, and axial members that bend, in which case the equivalent rotational springs become distributed over the member length. As a final generalization, the equivalent springs are distributed over the full spatial domain, enabling the consideration of such structures as i) axial members with different lateral rotations over the cross-section, ii) bending members with varying direct and shear stresses, and iii) continuum systems such as plates and general solids.

An important feature of the proposed rotational spring analogy relates to the simple conceptual framework it furnishes, which sheds new light on geometric nonlinearity in general, and buckling analysis in particular. Within this framework, buckling can be conceived as the result of a sufficiently destabilizing geometric stiffness, arising from negative equivalent rotational springs, which can overcome the stabilizing structural stiffness, arising typically from the material response, in a mode of deformation representing the buckling mode. The applicability of the proposed analogy is discussed for SDOF, MDOF and continuous problems, where for the latter two cases the computational benefit of problemreduction using one or more assumed modes is demonstrated. Within this context, it is shown 
that important buckling issues, such as i) the influence of initial prestress, ii) the interaction between different loads, and iii) the effect of transverse load location relative to the axis of a beam, can be easily assessed in terms of the contribution of additional equivalent rotational springs to the geometric stiffness associated with the buckling mode under consideration.

Another related and significant aspect of the proposed analogy is its ability to explain the intricacies of previous approaches to buckling and geometrically nonlinear analysis. For example, it shows that the significance of the local P- $\delta$ effect in beam-column elements is entirely related to the relative magnitudes of local and chord rotations in the considered deformation mode, demonstrating further that convergence can be achieved with increasing elements even if the local P- $\delta$ effect were to be ignored. It also offers intuitive explanations to previous buckling formulae dealing with a range of structures, including beams, columns, arches, and plates.

Finally, the proposed rotational spring analogy presents a practical framework for buckling analysis, which overcomes the limitations of conventional methods based on equilibrium in the perturbed configuration, and which avoids the complexity of the more rigorous methods that require at least quadratic kinematic descriptions for the deformed configuration. Both advantages are demonstrated using a number of illustrative problems, which, to name but a few, include a rigid bar system with prestressed cables, a beam subject to lateral torsional buckling under an elevated point load, and an arch subject to planar buckling under different types of distributed load.

In view of its conceptual power and demonstrable practical benefits, the proposed rotational spring analogy can potentially play an important role in the teaching and understanding of buckling analysis, and can offer a simplified approach to the buckling assessment of various structural systems that is more amenable to design-oriented application. 


\section{REFERENCES}

[1] Allen, H.G., and Bulson, P.S. (1980), Background to Buckling, McGraw Hill.

[2] Bažant, Z.P., and Cedolin, L. (1991), Stability of Structures, Dover.

[3] Belytschko, T., Liu, W.K., and Moran, B. (2000), Nonlinear Finite Elements for Continua and Structures, Wiley.

[4] Bradford, M.A. (1992), "Lateral-Distortional Buckling of Steel I-Section Members", Journal of Constructional Steel Research, 23(1-3), 97-116.

[5] Chan, S.L., and Zhou, Z.H. (2000), "Non-linear Integrated Design and Analysis of Skeletal Structures by 1 Element per Member”, Engineering Structures, 22(3), 246-257.

[6] Crisfield, M.A. (1991), Nonlinear Analysis of Solids and Structures, Vol. 1, Wiley.

[7] Hodges, D.H. (1999), "Non-Linear Inplane Deformation and Buckling of Rings and High Arches", International Journal of Non-Linear Mechanics, 34, 723-737.

[8] Horne, M.R. (1975), “An Approximate Method for Calculating the Elastic Critical Loads of Multi-Storey Plane Frames", The Structural Engineer, 53(6), 242-248.

[9] Izzuddin, B.A. (1996), "Quartic Formulation for Elastic Beam-Columns Subject to Thermal Effects", Journal of Engineering Mechanics, ASCE, 122(9), 861-871.

[10] Izzuddin, B.A. (2001), "Conceptual Issues in Geometrically Nonlinear Analysis of 3D Framed Structures", Computer Methods in Applied Mechanics and Engineering, 191(8-10), 1029-1053.

[11] Izzuddin, B.A. (2005), “An Enhanced Co-rotational Approach for Large Displacement Analysis of Plates", International Journal for Numerical Methods in Engineering, 64(10), 1350-1374.

[12] Nethercot, D.A., and Rockey, K.C. (1971), “A Unified Approach to the Elastic Lateral Buckling of Beams", The Structural Engineer, 49(7), 321-330.

[13] Timoshenko, S.P., and Gere, J.M. (1961), Theory of Elastic Stability, McGraw Hill. 


\section{LIST OF FIGURES}

Figure 1. Equivalent rotational spring for geometric stiffness of straight element

Figure 2. Equivalent rotational spring for geometric stiffness of bending element

Figure 3. Reduction of local geometric nonlinearity with number of elements

Figure 4. Equivalent rotational spring for geometric stiffness of radial load

Figure 5. Axial member with flexible lateral restraint

Figure 6. Rigid bar system with prestressed cables

Figure 7. Cantilever truss with flexible lateral restraints

Figure 8. Frame subject to lateral buckling

Figure 9. Cantilever column with flexible support

Figure 10. Buckling load factor for cantilever column with flexible support

Figure 11. Circular arch subject to distributed load

Figure 12. Buckling distributed load for circular arch

Figure 13. Column subject to torsional buckling

Figure 14. Beam subject to lateral torsional buckling under midspan point load 

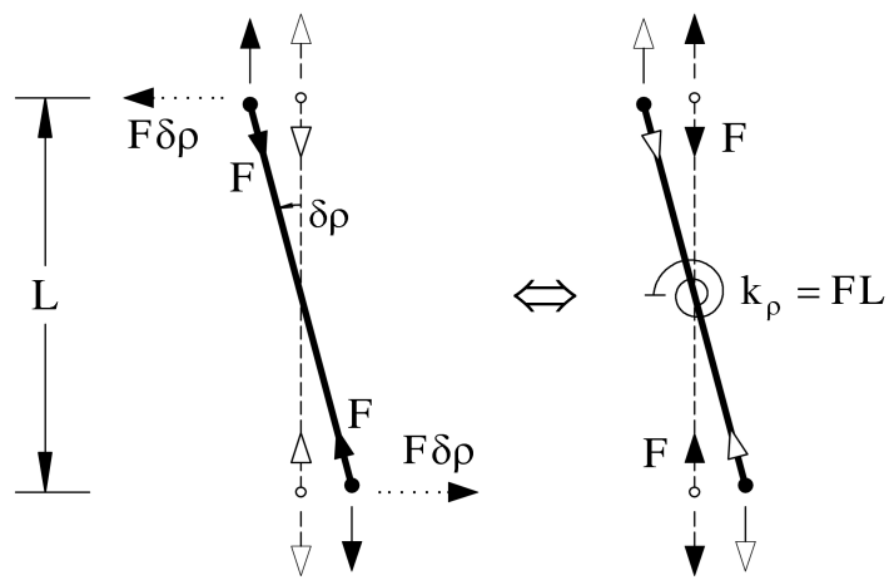

Figure 1. Equivalent rotational spring for geometric stiffness of straight element 


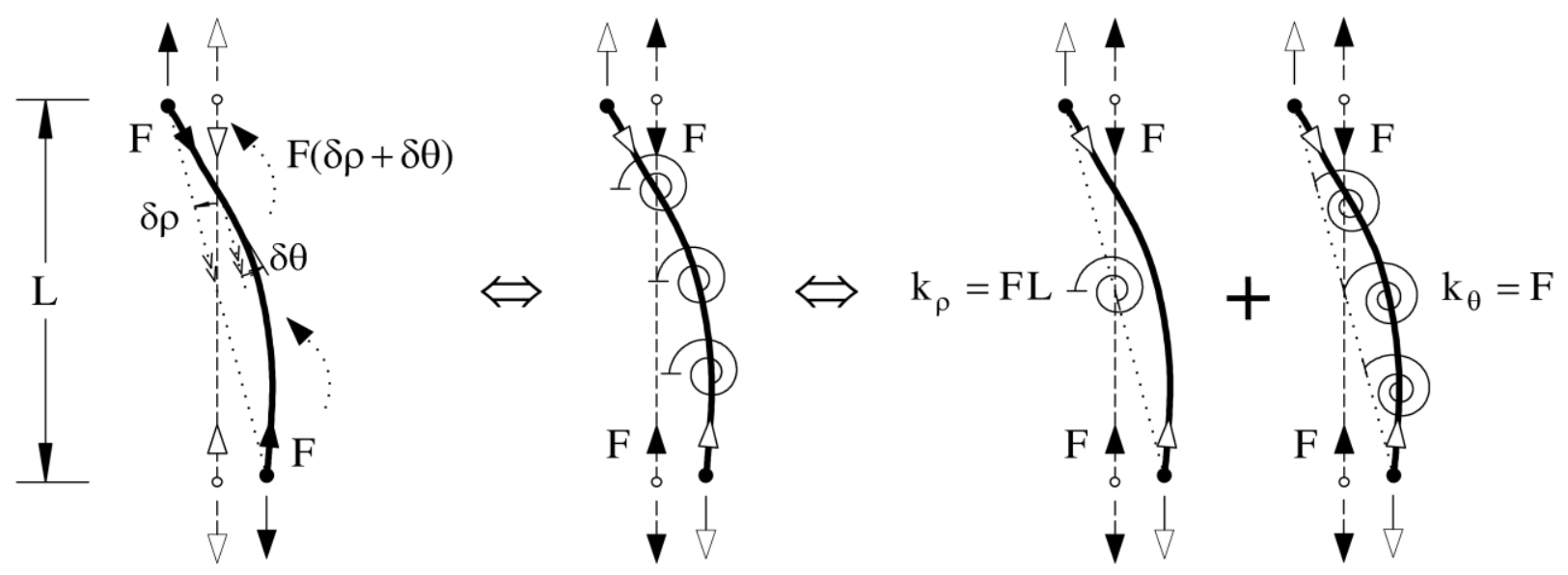

Figure 2. Equivalent rotational spring for geometric stiffness of bending element 


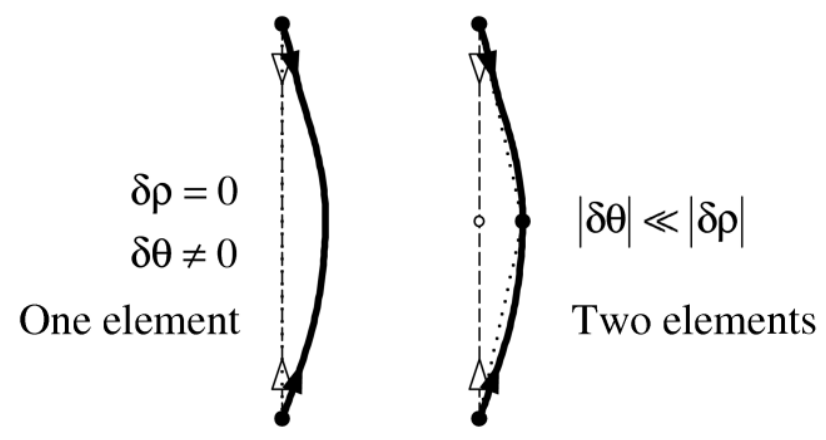

Figure 3. Reduction of local geometric nonlinearity with number of elements

Izzuddin: Rotational Spring Analogy for Buckling Analysis 


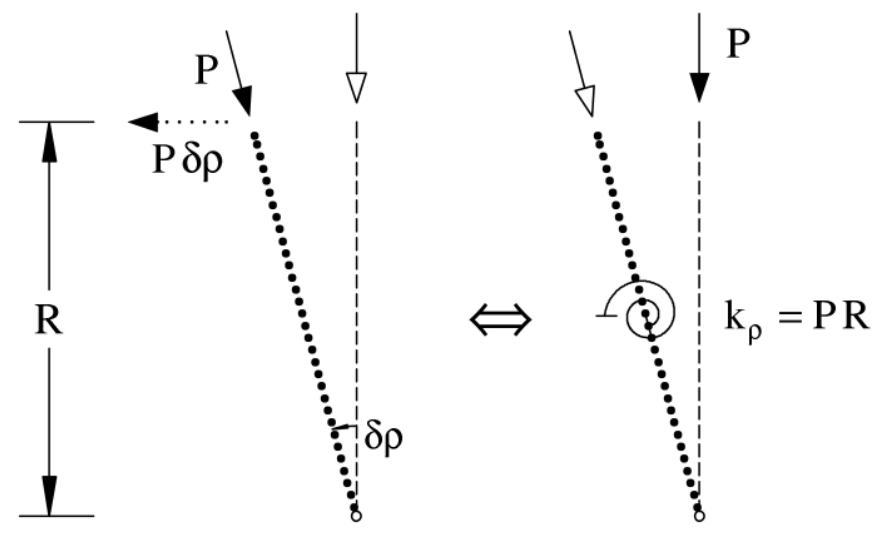

Figure 4. Equivalent rotational spring for geometric stiffness of radial load 
\begin{tabular}{l|l|}
$\otimes \begin{array}{l}\text { Equivalent rotational } \\
\text { springs }\end{array}$ & $\boldsymbol{\rho}_{1}=-\rho_{2}=\mathrm{U} / \mathrm{L}$ \\
$\mathbf{k}_{\mathrm{\rho} 1}=\mathbf{k}_{\rho 2}=-\mathrm{PL}$
\end{tabular}

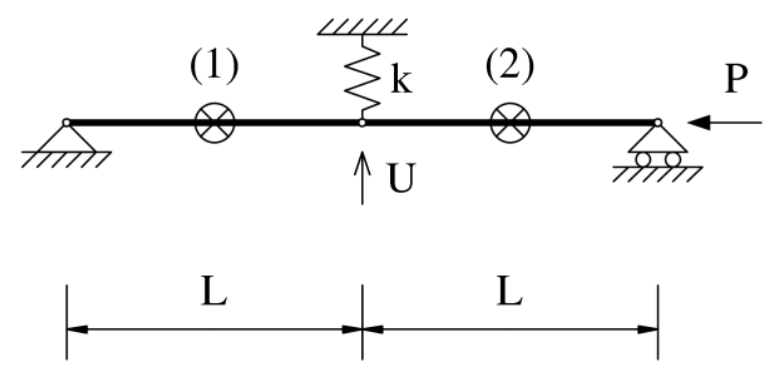

Figure 5. Axial member with flexible lateral restraint

Izzuddin: Rotational Spring Analogy for Buckling Analysis 


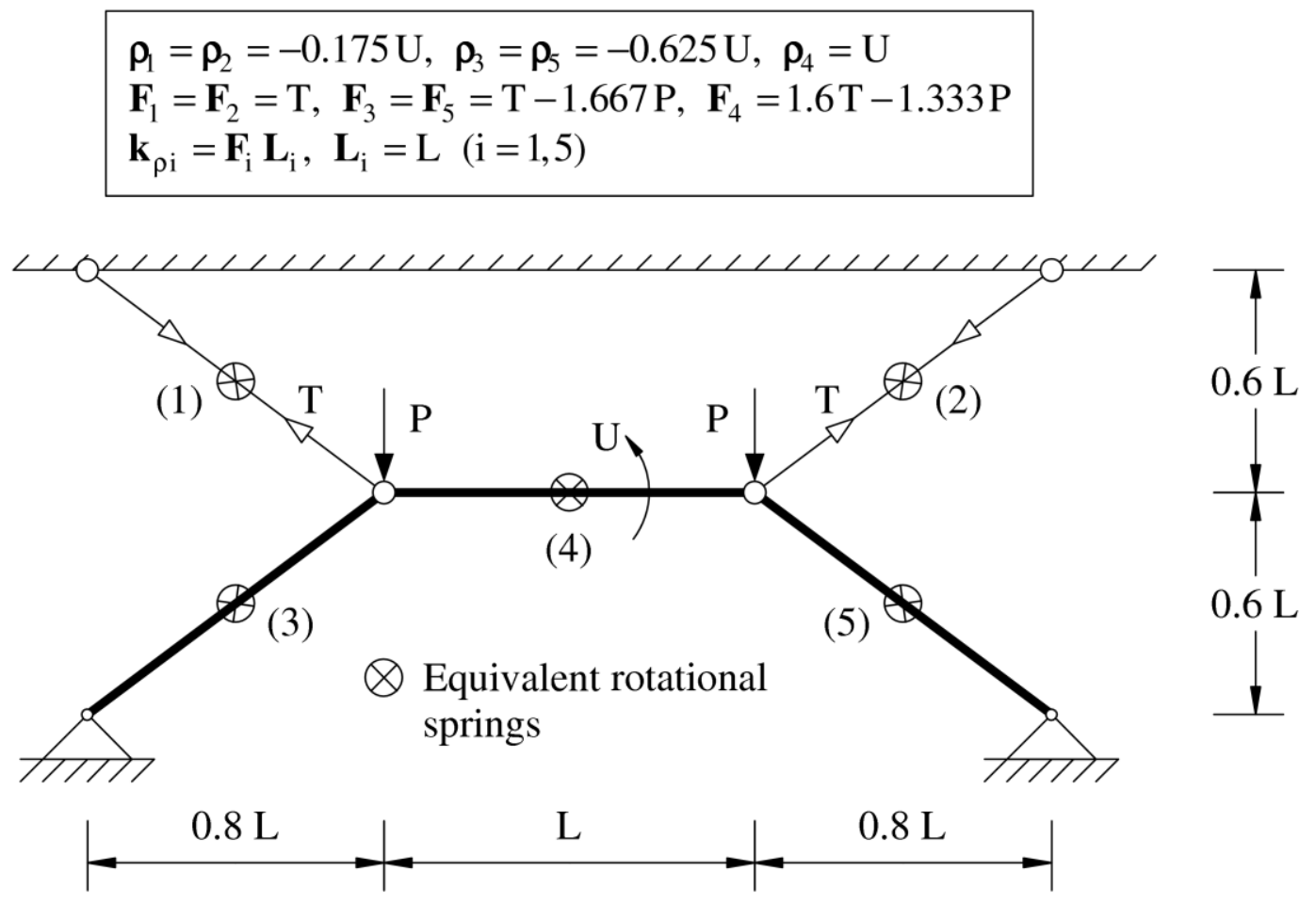

Figure 6. Rigid bar system with prestressed cables 


$$
\mathbf{T}_{\rho}=\frac{1}{\mathrm{~L}}\left[\begin{array}{cc}
0.8333 & 0 \\
0 & 0.8333 \\
2 & -2 \\
0.7692 & 0 \\
0 & 0.7692
\end{array}\right], \quad \mathbf{F}=\left\{\begin{array}{c}
-0.5 \\
0.5 \\
0.20833 \\
-0.54167 \\
-0.54167
\end{array}\right\} \mathrm{P}+\left\{\begin{array}{c}
1.2 \\
-1.2 \\
-0.5 \\
-1.3 \\
1.3
\end{array}\right\} \mathrm{Q}, \quad \mathbf{L}=\left\{\begin{array}{l}
1.2 \\
1.2 \\
0.5 \\
1.3 \\
1.3
\end{array}\right\} \mathrm{L}
$$

$\otimes$ Equivalent rotational springs
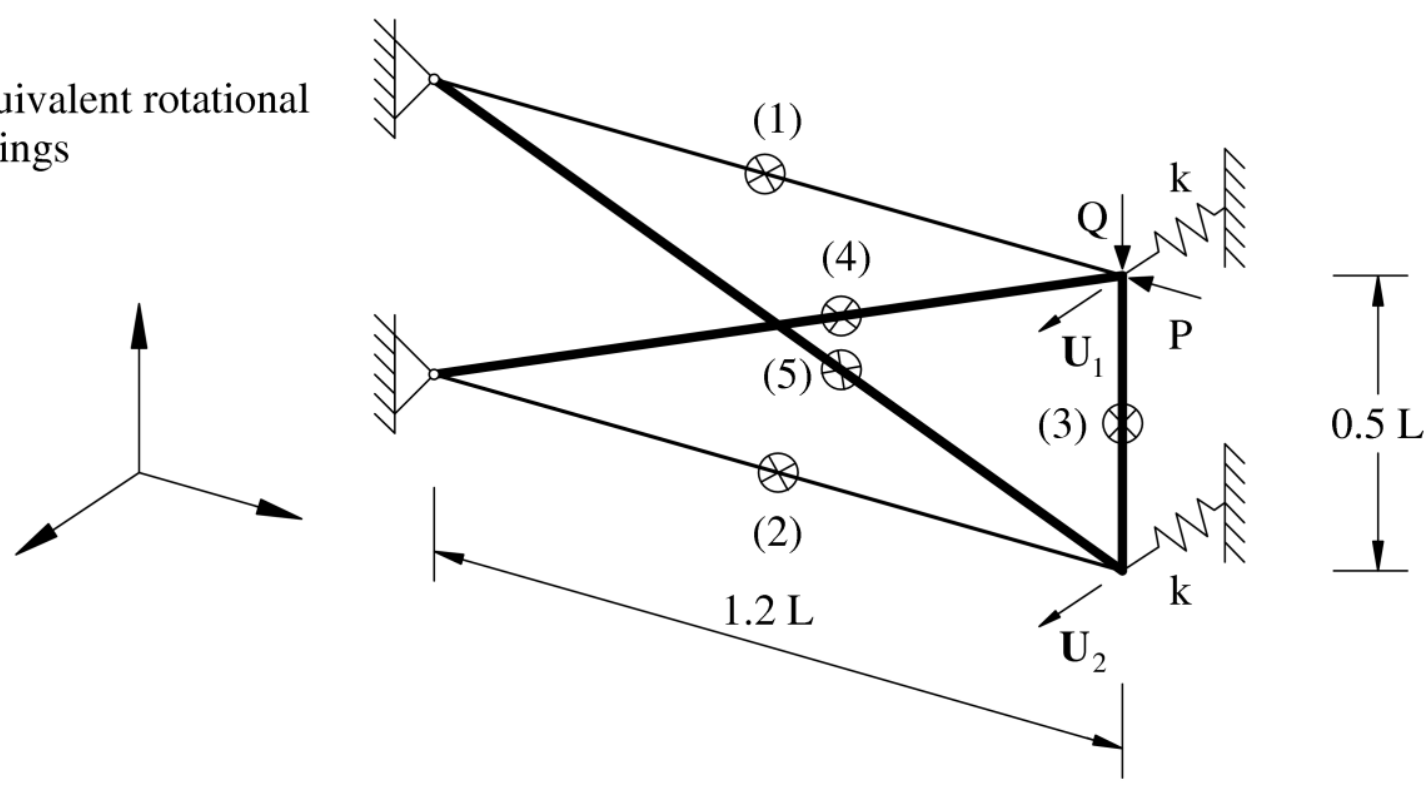

Figure 7. Cantilever truss with flexible lateral restraints 


$$
\begin{aligned}
& \mathbf{Q}=\left\{\begin{array}{c}
0.5 \\
1 \\
0.5
\end{array}\right\} \mathrm{ku}, \quad \mathbf{U}=\left\{\begin{array}{c}
0.25 \\
0.75 \\
0.25
\end{array}\right\} \mathrm{u}, \quad \boldsymbol{\rho}=\left\{\begin{array}{c}
0.25 / \mathrm{L} \\
0.5 / \mathrm{L} \\
-0.5 / \mathrm{L} \\
-0.25 / \mathrm{L}
\end{array}\right\} \mathrm{u} \\
& \mathbf{L}_{\mathrm{i}}=\mathrm{L}, \quad \mathbf{F}_{\mathrm{i}}=-\mathrm{P}, \quad \mathbf{k}_{\rho \mathrm{i}}=\mathbf{F}_{\mathrm{i}} \mathbf{L}_{\mathrm{i}} \quad(\mathrm{i}=1,4)
\end{aligned}
$$

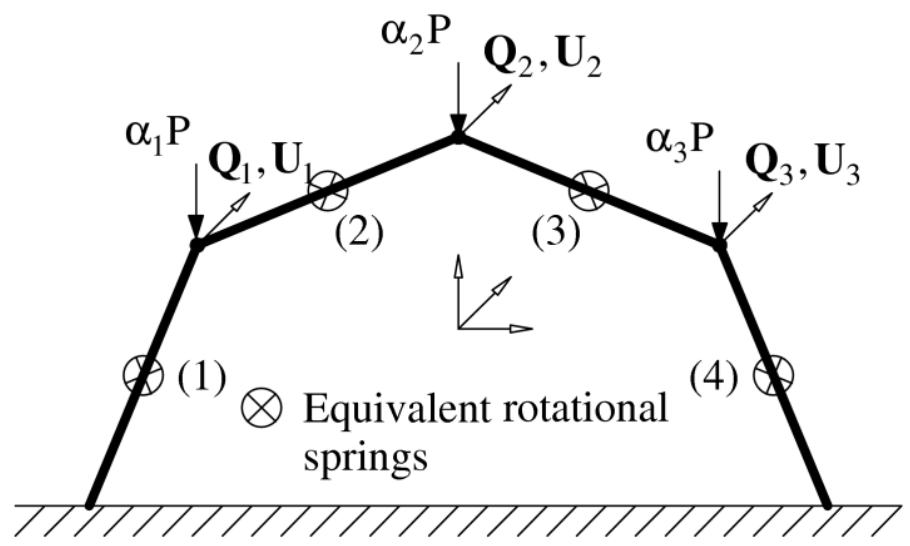

Figure 8. Frame subject to lateral buckling 


$$
\rho=\mathbf{U}_{1}, \quad v=\mathbf{U}_{2} L\left[\frac{\mathrm{x}}{\mathrm{L}}-\frac{3}{2}\left(\frac{\mathrm{x}}{\mathrm{L}}\right)^{2}+\frac{1}{2}\left(\frac{\mathrm{x}}{\mathrm{L}}\right)^{3}\right], \quad \theta=\frac{\mathrm{dv}}{\mathrm{dx}}=\mathbf{U}_{2}\left[1-3 \frac{\mathrm{x}}{\mathrm{L}}+\frac{3}{2}\left(\frac{\mathrm{x}}{\mathrm{L}}\right)^{2}\right]
$$

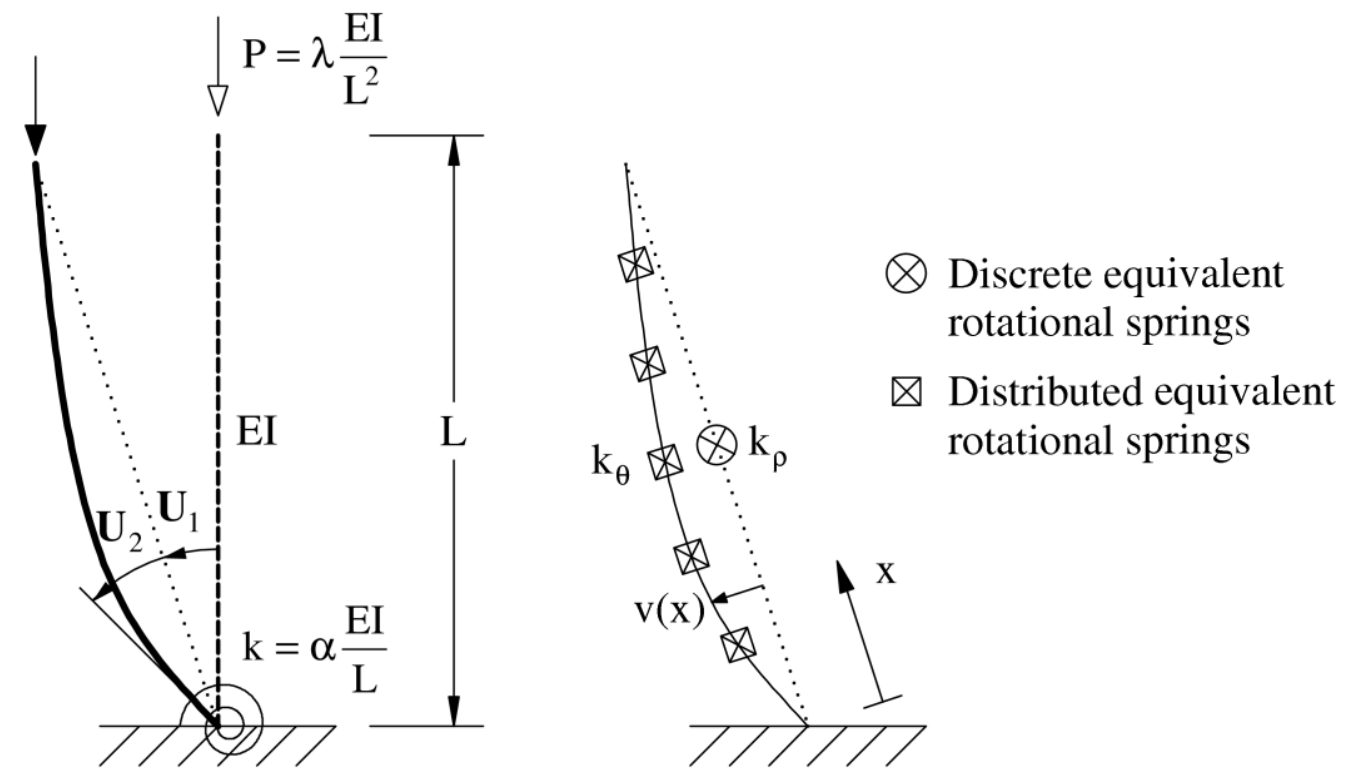

Figure 9. Cantilever column with flexible support 


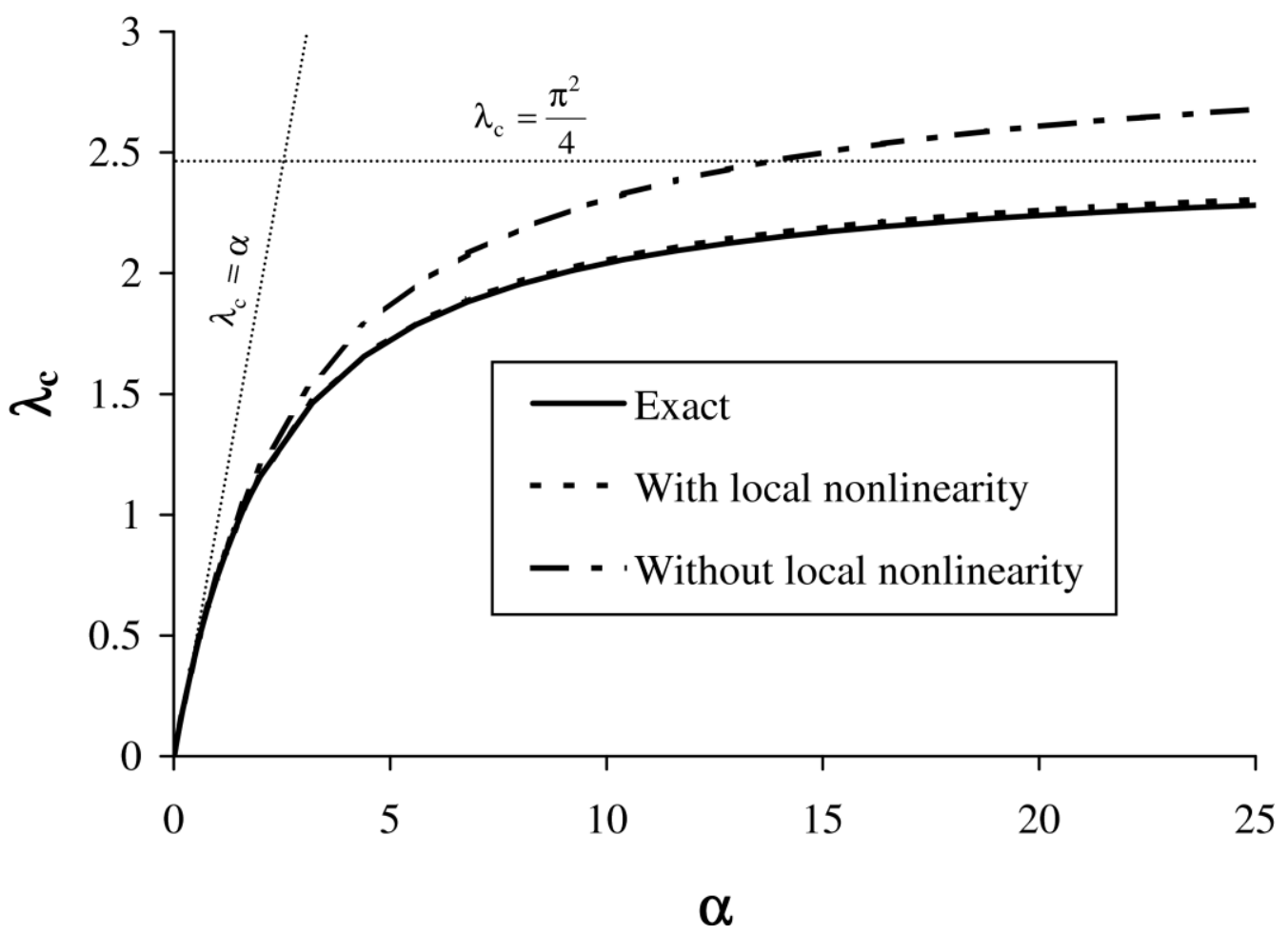

Figure 10. Buckling load factor for cantilever column with flexible support 

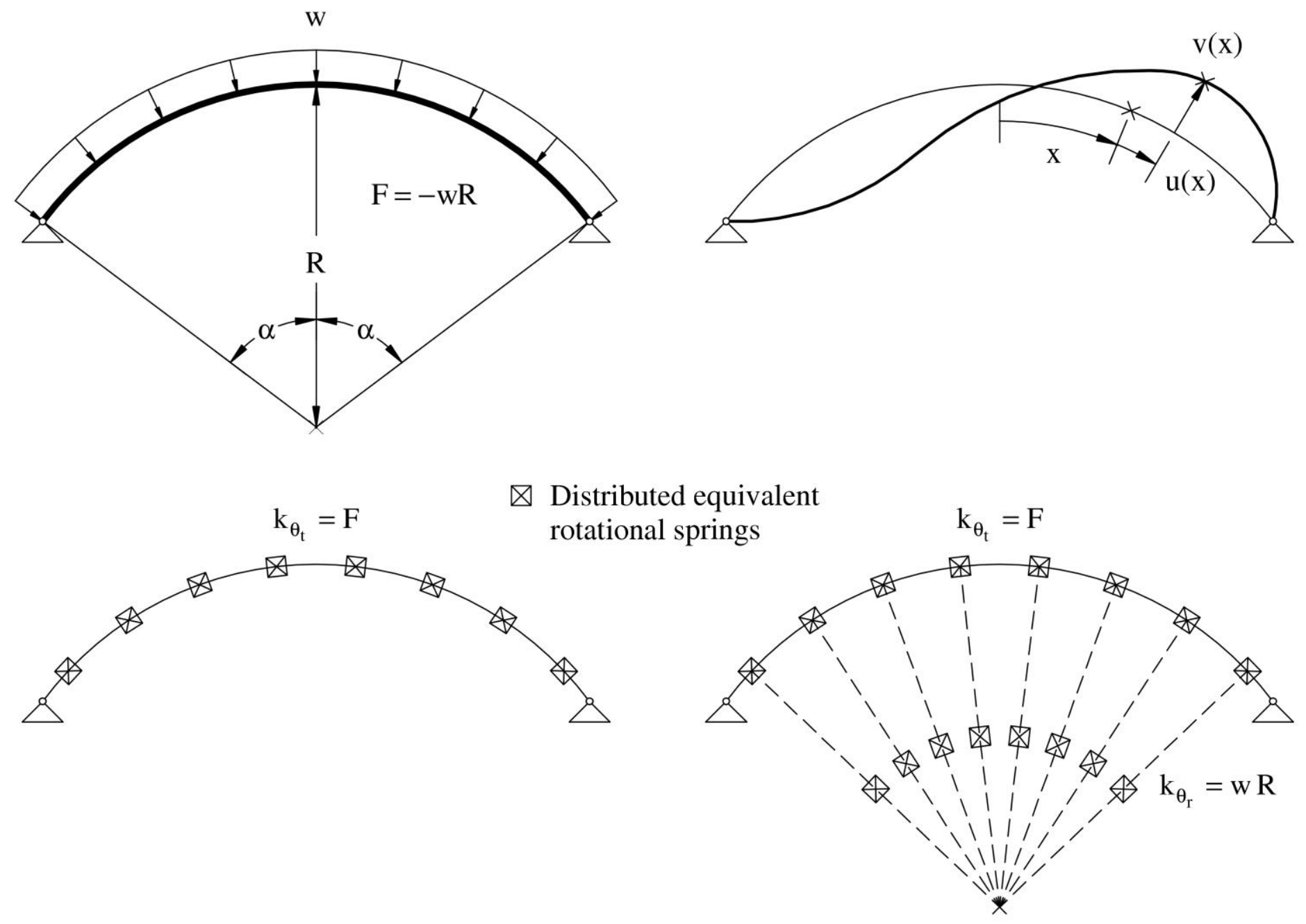

Fixed load direction

Radial load direction

Figure 11. Circular arch subject to distributed load 


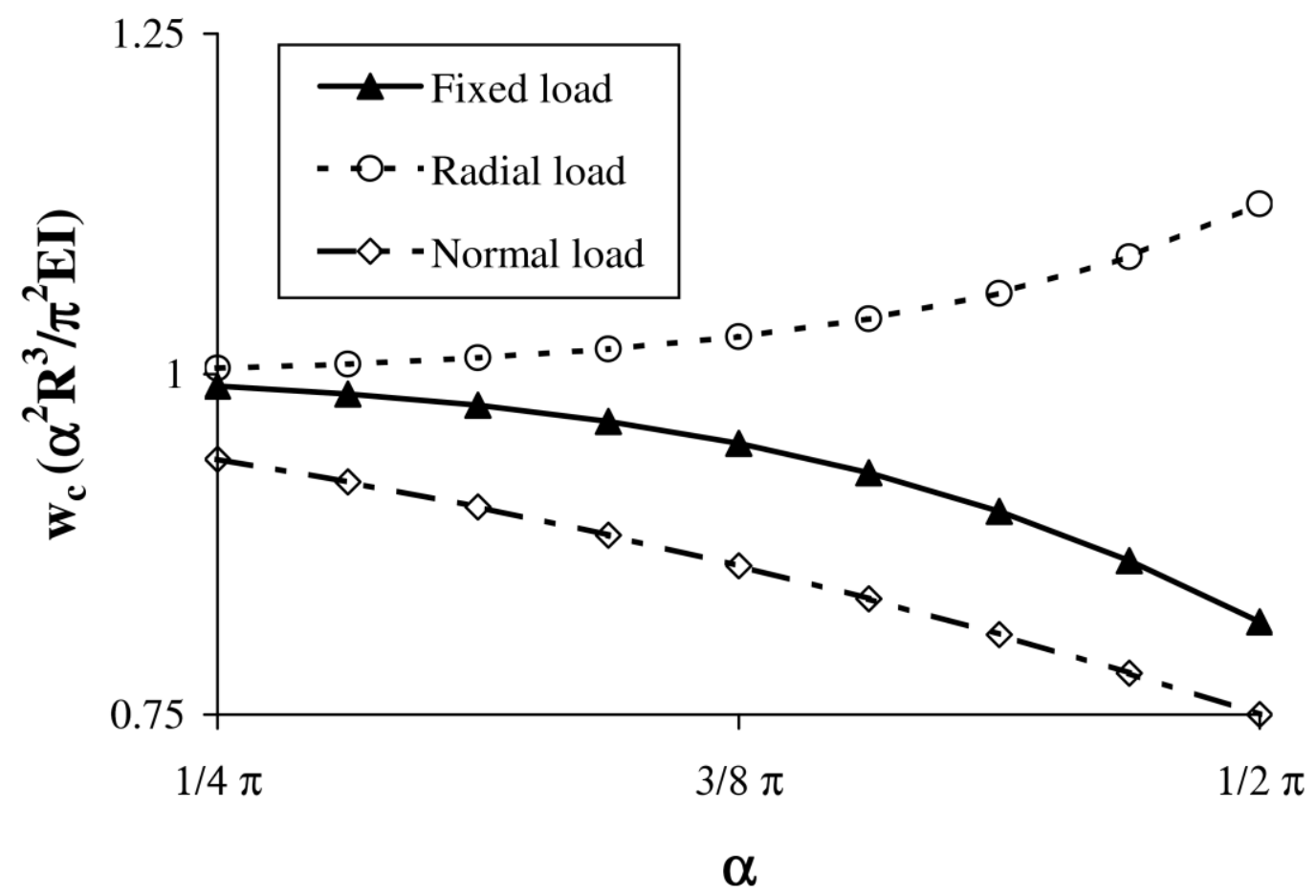

Figure 12. Buckling distributed load for circular arch 

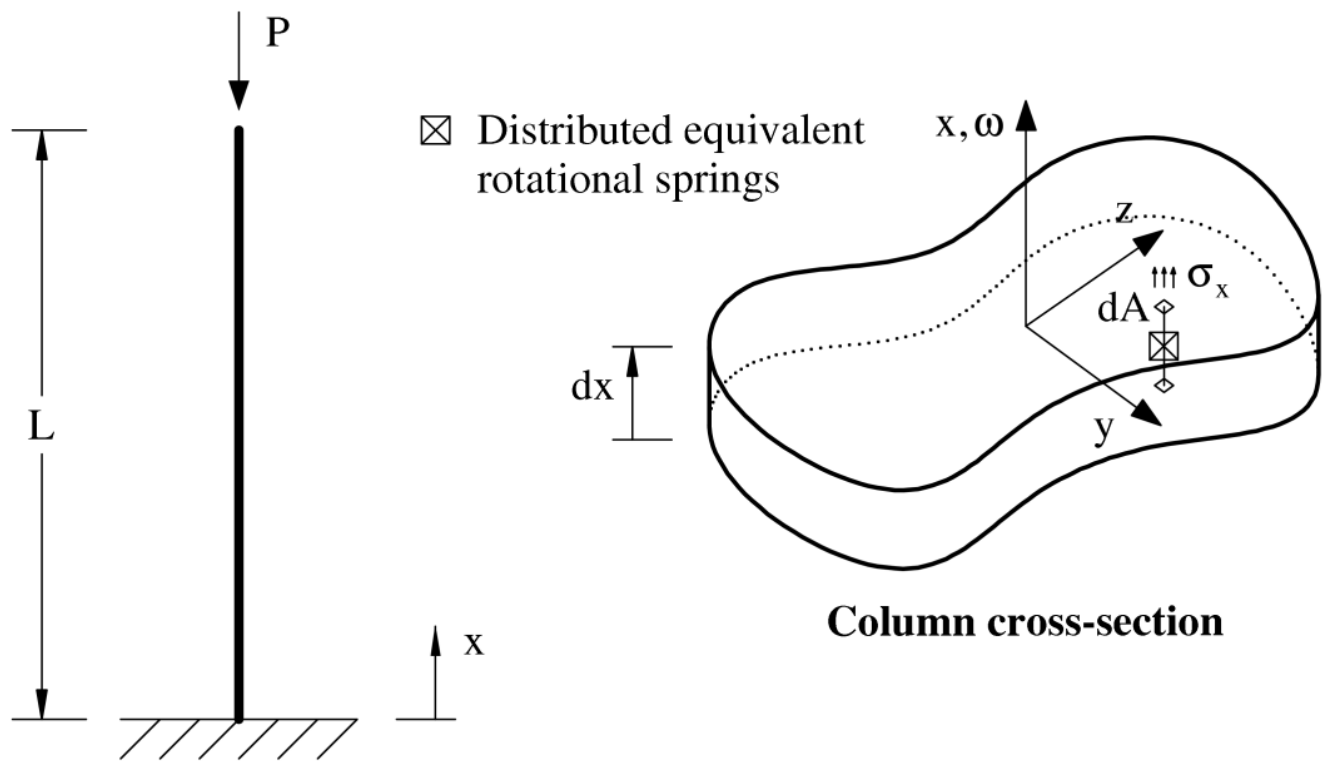

Column cross-section

Figure 13. Column subject to torsional buckling

Izzuddin: Rotational Spring Analogy for Buckling Analysis 


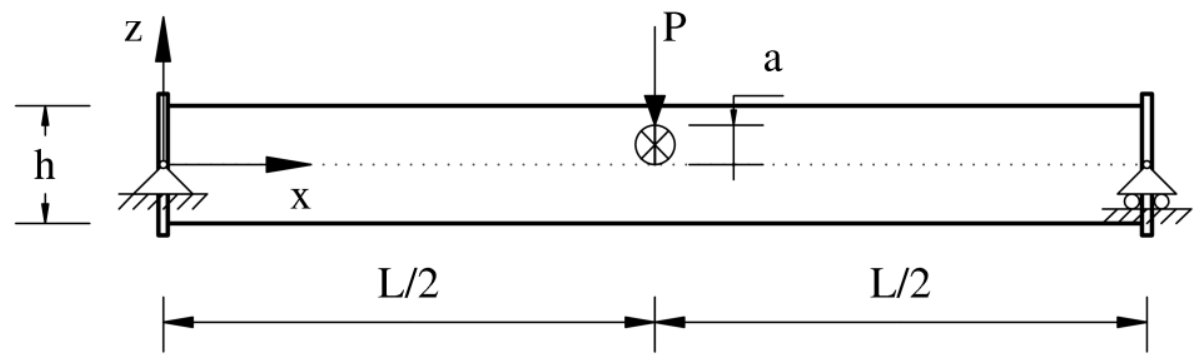

$\otimes$ Discrete equivalent rotational spring

$\otimes$ Distributed equivalent rotational springs
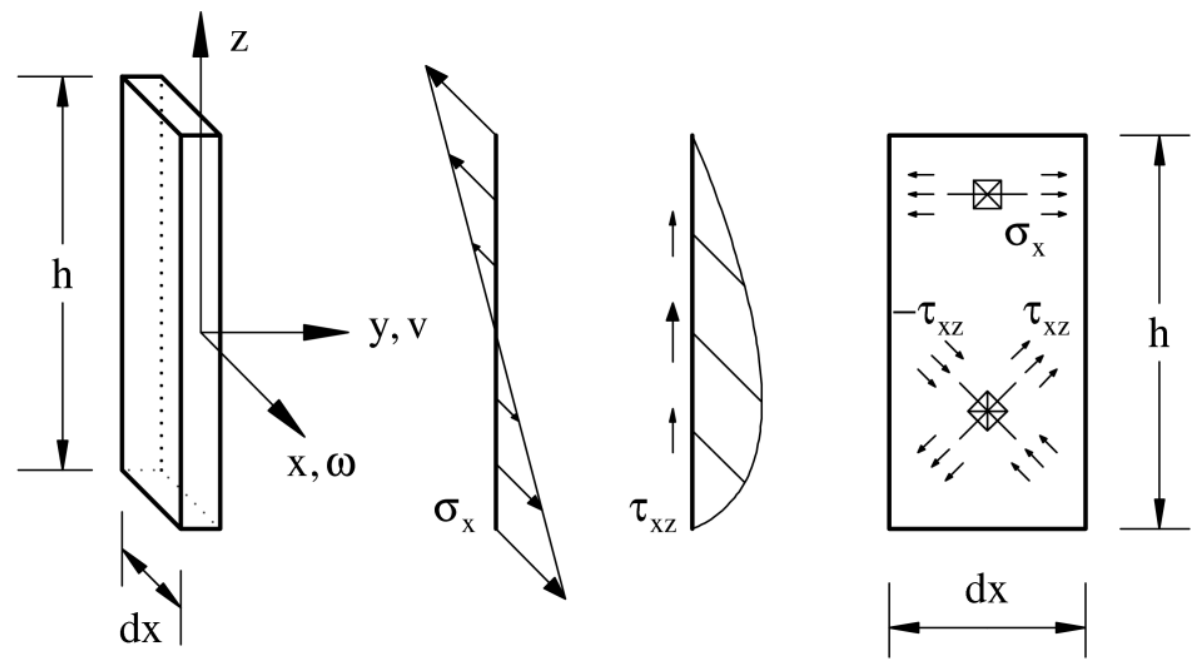

Figure 14. Beam subject to lateral torsional buckling under midspan point load 